\title{
As emoções à flor da pele e seus possíveis manejos na pandemia da COVID-19
}

\author{
Emotions on the surface and their possible management in the COVID-19 pandemic \\ Emociones en la superficie y su posible manejo en la pandemia de COVID-19
}

Recebido: 19/07/2021 | Revisado: 23/07/2021 | Aceito: 27/07/2021 | Publicado: 04/08/2021

\author{
Beatriz Soares da Silva \\ ORCID: https://orcid.org/0000-0002-6205-9759 \\ Universidade Federal do Rio de Janeiro, Brasil \\ E-mail: biass.soares@gmail.com \\ Paula Isabella Marujo Nunes da Fonseca \\ ORCID: https://orcid.org/0000-0002-8524-0147 \\ Universidade Federal do Rio de Janeiro, Brasil \\ E-mail: paulaisabellamarujo@gmail.com \\ Patrícia Duarte da Silva \\ ORCID: https://orcid.org/0000-0002-6888-2762 \\ Universidade Federal do Rio de Janeiro, Brasil \\ E-mail:pattyduartesilva@gmail.com
}

\begin{abstract}
Resumo
Objetivos: identificar as principais emoções vivenciadas pela sociedade no período da pandemia da COVID-19 e descrever o manejo das emoções pela sociedade no período da COVID-19. Método: revisão integrativa, com coleta de dados realizada em maio 2020 e fevereiro 2021. As buscas de artigos foram realizadas a partir do portal Periódicos CAPES, nas bases de dados MEDLINE COMPLETE; SCIELO; LILACS, e Google Scholar, sendo buscados nos idiomas Português, Inglês e Espanhol. As frases de busca estruturadas foram "emoções" and "pandemia"; "saúde mental" and "pandemia"; "pandemia" and "autoconhecimento". Para serem incluídos na pesquisa os materiais deveriam ser artigos, manuais, editoriais disponíveis na íntegra e gratuitamente online. Já as teses, dissertações, monografias e livros se enquadram nos critérios de exclusão. Resultados: Selecionados 144 os quais demonstram que as emoções mais vivenciadas durante a pandemia são: medo,tristeza e raiva, sendo estas acompanhadas por características de emoções como a ansiedade, frustração, solidão e angústia. Ademais, os estudos também indicam medidas de enfrentamento para lidar com as emoções e sentimentos a fim de buscar a melhor maneira de passar por esse período, assim como emergiram também, caminhos para autoconhecimento e desenvolvimento da inteligência emocional. Conclusão: O estudo contribui com os achados para a área de saúde mental, pois diante das principais emoções vivenciadas pela sociedade no período da pandemia da COVID-19 e de seus manejos, foi possível visualizar objetivamente os caminhos exitosos percorridos pela sociedade para o enfrentamento mais salutar dos desafios impostos pela atual pandemia.
\end{abstract}

Palavras-chave: Emoções; Saúde mental; COVID-19.

\begin{abstract}
Objectives: to identify the main emotions experienced by society during the period of the COVID-19 pandemic and to describe the management of emotions by society during the period of COVID-19. Method: integrative review, with data collection carried out in May 2020 and February 2021. articles were carried out from the CAPES Periodicals portal, in the MEDLINE COMPLETE databases; SCIELO; LILACS, and Google Scholar, being searched in Portuguese, English and Spanish. The structured search phrases were "emotions" and "pandemic" ; "mental health" and "pandemic" ; "pandemic" and "self-knowledge". To be included in the research, materials should be articles, manuals, editorials available in full and free online. Theses, dissertations, monographs and books, on the other hand, meet the exclusion criteria. Results: 144 were selected which demonstrate that the most experienced emotions during the pandemic are: fear, sadness and anger, which are accompanied by characteristics of emotions such as anxiety, frustration, loneliness and anguish. Furthermore, studies also indicate coping measures to deal with emotions and feelings in order to find the best way to go through this period, as well as emerging paths for self-knowledge and development of emotional intelligence. Conclusion: The study contributes to the findings for the area of mental health, because given the main emotions experienced by society during the period of the COVID-19 pandemic and its management, it was possible to objectively visualize the successful paths taken by society for a more healthy coping challenges posed by the current pandemic.
\end{abstract}

Keywords: Emotions; Mental health; COVID-19.

\section{Resumen}

Objetivos: identificar las principales emociones vividas por la sociedad durante el período de la pandemia de COVID19 y describir el manejo de las emociones por parte de la sociedad durante el período de COVID-19. Método: revisión 
integradora, con recolección de datos realizada en mayo de 2020 y febrero de 2021. Los artículos se realizaron desde el portal de publicaciones periódicas CAPES, en las bases de datos MEDLINE COMPLETE; SCIELO; LILACS y Google Scholar, en portugués, inglés y español. Las frases de búsqueda estructuradas fueron "emociones" y "pandemia"; "salud mental" y "pandemia", "pandemia" y "autoconocimiento". Para ser incluido en la investigación, los materiales deben ser artículos, manuales, editoriales disponibles en línea completa y gratuita. Tesis, disertaciones, monografías y libros, en cambio, cumplen con los criterios de exclusión. Resultados: Se seleccionaron 144 que demuestran que las emociones más vividas durante la pandemia son: miedo, tristeza e ira, las cuales se acompañan de características de las emociones como ansiedad, frustración, soledad y angustia. Además, los estudios también señalan medidas de afrontamiento para afrontar las emociones y los sentimientos con el fin de encontrar la mejor forma de atravesar este período, así como también han surgido caminos para el autoconocimiento y el desarrollo de la inteligencia emocional. Conclusión: El estudio contribuye a los hallazgos para el área de salud mental, pues dadas las principales emociones vividas por la sociedad durante el período de la pandemia de COVID-19 y su manejo, fue posible visualizar objetivamente los caminos exitosos tomados por la sociedad para un afrontamiento más saludable de los desafíos planteados por la pandemia actual.

Palabras clave: Emociones; Salud mental; COVID-19.

\section{Introdução}

A COVID-19, doença causada pelo novo coronavírus (Sars-Cov-2), foi caracterizada pela Organização Mundial da Saúde como uma pandemia em 11 de março de 2020, alarmando a população mundial sobre o período difícil e incerto que estava iniciando. Anteriormente, a gravidade da doença já havia sido comunicada uma vez que em 30 de janeiro de 2020 , a OMS declarou que o surto do novo coronavírus constitui uma Emergência de Saúde Pública de Importância Internacional (ESPII) - o mais alto nível de alerta da Organização. Tendo isso em vista, foi comprovado que os países estavam diante de um vírus que apresenta alto risco para os serviços de saúde pública do mundo e que possui grande distribuição geográfica. (OPAS/OMS, 2021.)

Atrelado ao cenário de ameaça aos sistemas de saúde pública dos países, a população enfrenta um período de intenso medo, principalmente da morte. No dia 12 de fevereiro de 2021, a folha informativa da OPAS/OMS registrou dois milhões, trezentos e sessenta mil e duzentos e oitenta mortes, esses dados ainda apresentam alto índice de aumento diário, sem números significativos do avanço das campanhas de vacinação, o que contribui para permanência do temor coletivo ao vírus. Diante dessa situação de incertezas e medos constantes, a pandemia causa sequelas na saúde mental, ultrapassando até as mortes provocadas pela enfermidade. (Books, et al. apud Ribeiro, et al. 2020)

No Brasil, o cenário de aflições decorrentes da pandemia da COVID-19 apresenta mais estressores, visto que as ações do Governo Federal tentam minimizar a gravidade da pandemia da COVID-19 e se posicionam em desacordo com os protocolos de prevenção propostos pela ANVISA e OMS. O país apresenta mais de 10\% das mortes totais no mundo, somando duzentos e sessenta e oito mil trezentos e setenta mortes no dia 10 de março de 2021, sendo esperados valores ainda mais altos em decorrência das novas variantes que circulam no país. Somado a esse panorama, o Brasil se encontra numa crise econômica de grande magnitude, uma vez que a crise sanitária causada pelo alto índice de contaminações e mortes pela COVID-19, alinhado a medidas de isolamento social, como tentativas de diminuir a transmissão do coronavírus, têm impactos econômicos e sociais. (Magalhães \& Cardoso, 2020.)

Diante do panorama exposto, a população tem enfrentado diariamente diversas emoções, sensações e sentimentos como consequência do intenso número de notícias e novas informações alarmantes sobre a COVID-19. O medo da incerteza, morte, perda do emprego, mudanças drásticas no estilo de vida, isolamento, distanciamento físico, separação da família e amigos estão relacionados a riscos mentais de alto nível. Além do medo, raiva e tristeza também são respostas a perda da sensação de liberdade e negação da situação preocupante que o mundo vivencia (Jakovljevic, 2020). A situação também é desencadeante de insônia, pesadelos, sintomas de ansiedade generalizada, sintomas depressivos, o aparecimento de sintomas obsessivos (lavar as mãos e limpar frequentemente objetos de uso são relatados pessoais), sintomas de estresse pós-traumático e também aumento do uso de substâncias, especialmente álcool (Pérez, et al 2020.) 
Portanto, diante deste cenário de emergentes sinais emocionais advindos da população frente à situação pandêmica, pensou-se sobre a relevância da identificação das emoções vivenciadas pela sociedade, uma vez que a partir deste primeiro passo é que possível cogitarmos a construção da Inteligência Emocional, conforme afirma Goleman (2011, p.54): "A aptidão de auto consciência das emoções se apresenta como fundamento central, da inteligência emocional, tendo em vista que é apenas após a identificação da emoção que a melhor forma de lidar com ela pode ser avaliada."

A inteligência emocional consiste na capacidade de identificar, e reconhecer, nossas emoções e dos outros, assim como ter aptidões que incluem autocontrole, zelo e persistência, e a capacidade de automotivação. (Goleman, 2011.). Reunindo como os cinco domínios fundamentais: conhecer as próprias emoções; lidar com emoções; reconhecer emoções dos outros; lidar com relacionamentos e motivar-se (Salovey \& Mayer apud Goleman,2011).

Desta forma, o presente estudo justifica-se, pois o período de distanciamento social imposto pela pandemia da COVID19 tem aflorado muitas emoções e sentimentos, sendo assim a partir do conhecimento destas emoções vivenciadas pela sociedade é possível refletirmos sobre possíveis caminhos gerais para a construção da inteligência emocional, medida que representa, no atual panorama mundial, meio de promoção e manutenção da saúde mental das pessoas.

Ademais, o estudo contribui para que a população tenha acesso a diversas formas que auxiliam, de forma eficaz, a lidar com as emoções e experiências vividas durante o período pandêmico. É importante destacar que ao buscar maneiras de lidar com as emoções tem-se como proposta buscar o equilíbrio, a fim de proporcionar a melhor forma de enfrentar as situações da vida, e não a supressão das emoções (Goleman, 2011).

Isto posto, o estudo propõe como objetivos identificar as principais emoções vivenciadas pela sociedade no período da pandemia da COVID-19 e descrever o manejo das emoções pela sociedade no período da COVID-19; apontar caminhos que possibilitam a construção da inteligência emocional.

\section{Metodologia}

O estudo trata-se de estudo de abordagem qualitativa, natureza descritiva e exploratória, realizada por meio de uma revisão integrativa de literatura sobre as emoções e seus manejos durante o período da COVID-19. A revisão integrativa é a mais ampla abordagem dentre as revisões de literatura, o que permite gerar uma visão consistente sobre a teoria analisada, sendo construída a partir de seis fases: elaboração da pergunta norteadora, busca na literatura, coleta de dados, análise crítica dos estudos incluídos, discussão dos resultados e apresentação da revisão integrativa. Esse tipo de revisão de literatura garante seu impacto e contribuições uma vez que além de reunir os dados e desenvolver políticas e protocolos, também desenvolve uma análise crítica sobre os dados (Souza, et al, 2010).

A pesquisa se norteou a partir de duas perguntas formuladas a partir da combinação mnemônica PCC (P: Population; C:Concept; C: Context), em que: P= Sociedade; C= Emoções; C= Pandemia da COVID-19. Desta forma, para este fim foram estruturadas as seguintes perguntas: "Quais são as emoções vivenciadas pela sociedade no período da pandemia da COVID-19?" e "Como se dá o manejo das emoções vivenciadas pela sociedade no período da pandemia da COVID-19?".

A coleta de dados foi realizada em dois momentos durante o período da pandemia, a partir do Google Scholar e do portal Periódicos CAPES, nas bases de dados MEDLINE COMPLETE; SCIELO; LILACS. Em ambos períodos foram utilizados os mesmos critérios de inclusão e exclusão. Inicialmente, as buscas foram feitas em maio de 2020, e o segundo momento foi em fevereiro de 2021, sendo os artigos buscados nos idiomas Português, Inglês e Espanhol. Para serem incluídos na pesquisa os materiais deveriam ser artigos, manuais, editoriais disponíveis na íntegra e gratuitamente online. Já as teses, dissertações, monografias e livros se enquadram nos critérios de exclusão.

Os direcionadores de busca selecionados foram os descritores "emoções”, "pandemia", "saúde mental”, e a palavrachave "autoconhecimento", sendo estruturadas, utilizando o operador booleano AND, as frases de busca "emoções" and 
"pandemia"; "saúde mental" and "pandemia"; "pandemia" and "autoconhecimento". O período de busca foi delimitado no ano de 2020 .

A partir disto, abaixo segue a Figura 1 que traz o percurso metodológico estabelecido a partir do diagrama de fluxo PRISMA (Moher, et al, 2009).

Figura 1 - Diagrama de Fluxo PRISMA (Moher, et al, 2009).

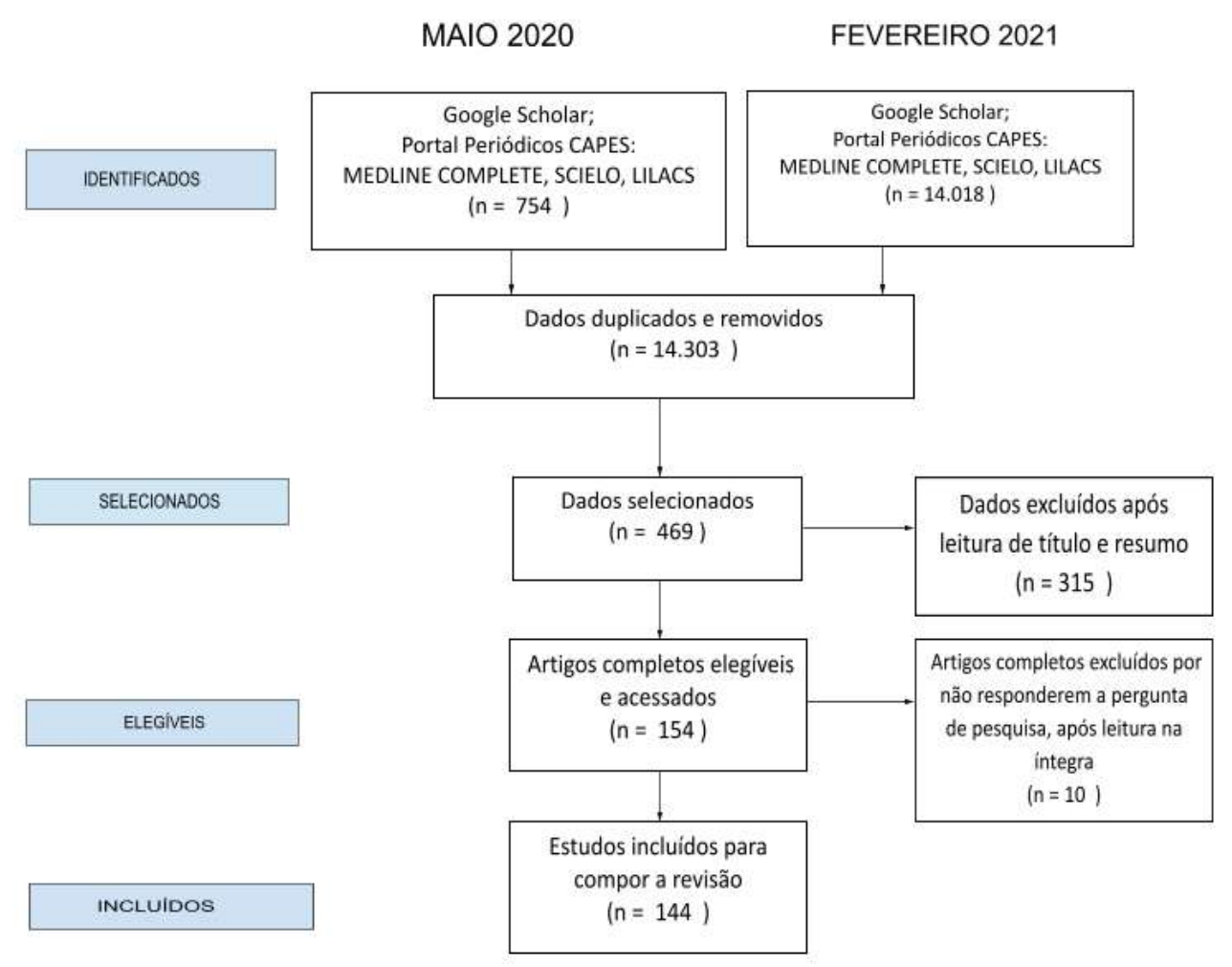

Fonte: Autores (2021).

Os estudos que atenderam aos critérios de inclusão foram analisados, inicialmente, a partir da leitura do título e resumo, a fim de identificar se respondiam às perguntas norteadoras da pesquisa. Os estudos que retornavam respostas às perguntas foram lidos na íntegra, com intuito de realizar uma análise mais profunda do conteúdo.

Utilizou-se a análise de conteúdo de Bardin (2011), que consiste num conjunto de técnicas de análises das comunicações, por procedimentos sistemáticos e objetivos, com intuito de obter os conteúdos das mensagens e indicadores que permitam a inferência dos conhecimentos. A análise de conteúdo é composta de três fases: pré-análise; exploração do material; tratamento dos resultados e interpretação.

Para organização e compilação dos dados, os artigos foram organizados destacando título, ano de publicação e revista, objetivo do artigo, público alvo, emoções identificadas e manejos das emoções encontrados. A partir desta etapa, foram identificadas as unidades de registro e a partir da sinalização das mesmas foram criadas categorias de sentido, distribuídas de modo a atender aos objetivos do estudo.

\section{Resultados}

Os 144 artigos selecionados foram publicados online no ano de 2020, sendo os artigos internacionais os mais publicados sobre o tema, totalizando 79 artigos incluídos (55\%), já os nacionais totalizaram 65 publicações (45\%). Os artigos foram 
publicados por 89 periódicos diferentes, dos quais se destacou a revista internacional Research, Society and Development com oito publicações (5\%) (Pereira, et al, 2020; Prigol \& Santos, 2020; Neves, et al, 2020; Rodrigues \& Lins, 2020; Santos, et al, 2020; Pimentel \& Silva, 2020; Silva et al, 2020; Dias, et al, 2020) e a nacional Revista Brasileira de Atividade Física com três publicações (2\%) (Rocha, et al, 2020; Gonçalvez, et al, 2020; Corrêa, et al, 2020.). Em relação às bases de dados, a MEDLINE COMPLETE foi a que retornou mais resultados à busca, sendo incluídos 59 artigos dessa base (41\%).

A partir do corpus de resultados, emergiu-se as seguintes categorias: Emoções à flor da pele na pandemia da COVID19; O manejo das emoções frente ao novo normal; e, O autoconhecimento como caminho para a construção da Inteligência Emocional - atalhos e desvios.

Com relação às emoções, tomar-se-á como base as definições e classificações utilizadas por Goleman (2011) e Casassus (2009). De acordo com Goleman (2011, p. 312) "as emoções se referem a um sentimento, seus pensamentos distintos e uma gama de tendências para agir." O autor considera como emoções: ira, tristeza, medo, prazer, amor, surpresa, nojo e vergonha. Casassus (2009, p.95-96), caracteriza como "emoções básicas aquelas que são biologicamente primitivas, não havendo necessidade que haja uma causa conhecida ou objeto para que ela ocorra.” Já as características de emoções, que também são chamadas de emoções secundárias ou derivadas, necessitam de desenvolvimento cognitivo e de autorreferência. As emoções e características de emoções, consideradas por este autor, são: Raiva - agressão, repugnância, ira, fúria, ódio; Tristeza - aflição, pesar, pessimismo, depressão; Medo - angústia, inquietação, horror, ansiedade, apreensão; Alegria - riso, felicidade; Amor amizade, afetividade, confiança.

Em relação às emoções vivenciadas e as características de emoções, que estão definidas de acordo com as referências de Inteligência Emocional (Goleman, 2011) e Educação Emocional (Casassus, 2009), não foram observadas distinções significativas entre as mais prevalentes no grupo de profissionais da saúde e sociedade. Na Figura 1, está a frequência de aparecimento das emoções nos estudos analisados. A Figura 2, traz as características de emoções que foram relatadas.

Figura 2 - Frequência das principais emoções vivenciadas pela sociedade em geral e profissionais da saúde.

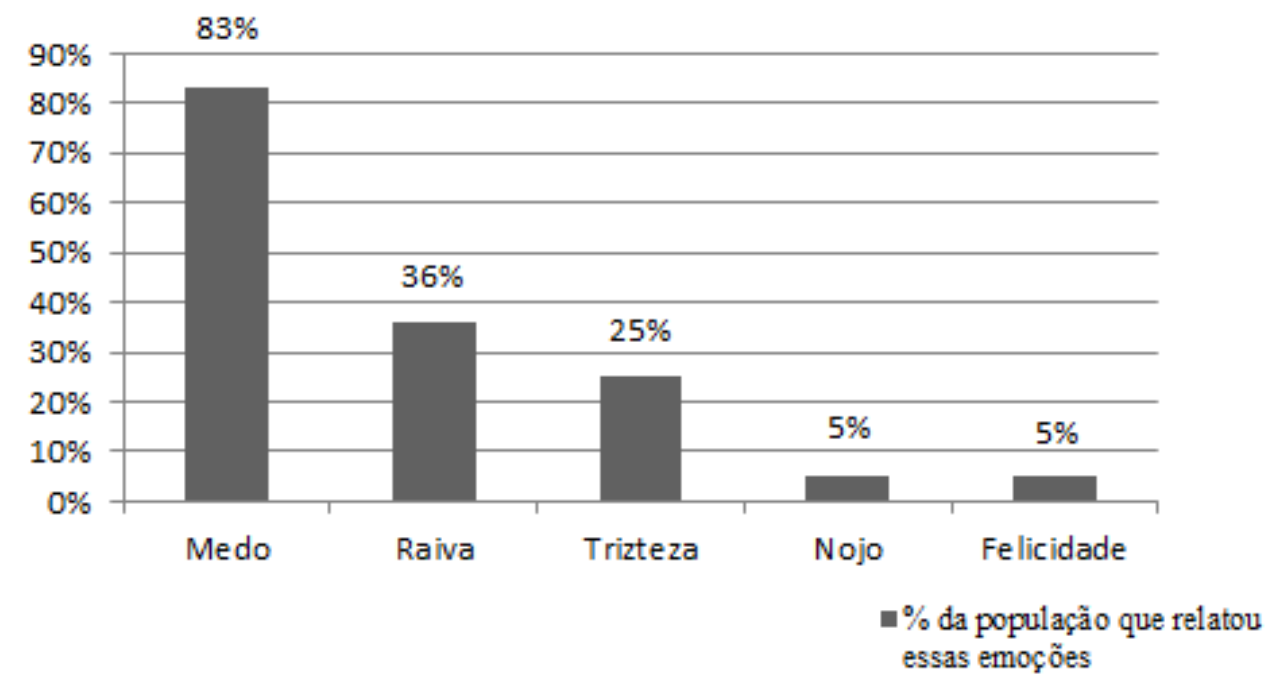

Fonte: Autores (2021). 
Figura 3 - Frequência das principais características das emoções vivenciadas pela sociedade em geral e profissionais da saúde.

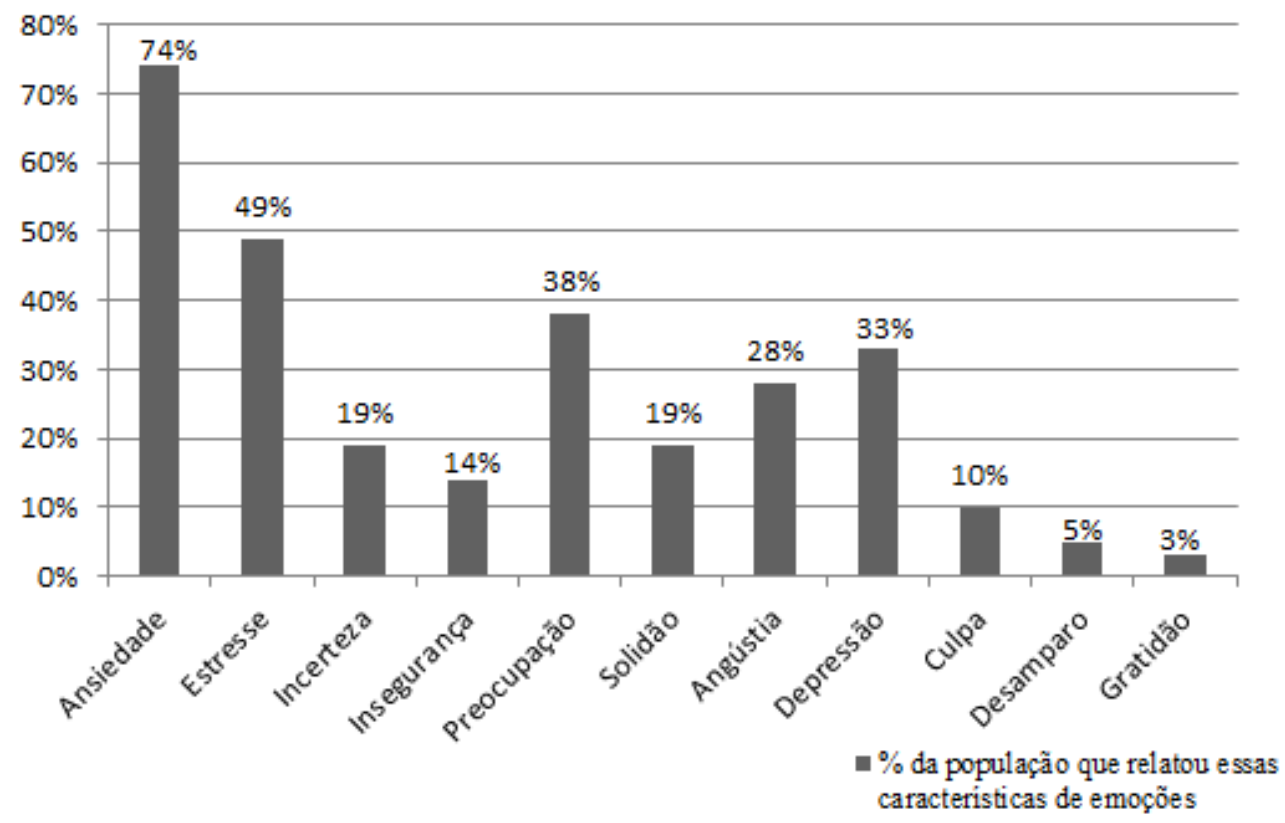

Fonte: Autores (2021).

Quanto aos manejos das emoções vivenciadas, 73 estudos (51\%) abordaram os manejos das emoções dos profissionais da saúde, que estão listados no Quadro 1. Já os manejos das emoções da sociedade, foram discutidos em 82 estudos (57\%), sendo estes destacados no Quadro 2. 
Quadro 1 - Principais aspectos relacionados ao manejo das emoções por profissionais de saúde na pandemia do Novo Coronavírus, 2021.

\begin{tabular}{|c|c|c|}
\hline $\begin{array}{l}\text { Aspectos relacionados } \\
\mathbf{a}(\mathbf{s}) / \mathbf{a o}(\mathbf{s})\end{array}$ & Manejos & Referências \\
\hline \multirow[t]{4}{*}{$\begin{array}{l}\text { Cuidados psicológicos e } \\
\text { emocionais oferecidos aos } \\
\text { profissionais }\end{array}$} & $\begin{array}{l}\text { Psicoterapia, Assistência Psicológica de } \\
\text { qualidade oferecida no trabalho, Receber } \\
\text { Apoio Psicológico/Online }\end{array}$ & $\begin{array}{c}\text { AA,AF,AG,AH,AK,AO,AQ,AR,AZ,BF,BJ,CA, } \\
\text { CB,CG,CS,CU,CX,DK,EE,FD,FH,CM,DE,DI,D } \\
\text { N,EC,EG,FA,DS, EH,EX,ED,DY,EL,CK }\end{array}$ \\
\hline & $\begin{array}{l}\text { Implementação Precoce de Apoio } \\
\text { Psicológico }\end{array}$ & $\mathrm{AB}, \mathrm{AM}, \mathrm{CF}, \mathrm{DJ}, \mathrm{DL}$ \\
\hline & $\begin{array}{l}\text { Oferta de canais de escuta das emoções, } \\
\text { Telemonitoramento em Saúde Mental para } \\
\text { promover Escuta e Acolhimento }\end{array}$ & CH,CU,CW,CX,CY,DQ,EC,EE,FD \\
\hline & $\begin{array}{l}\text { Serviços de Escuta com Profissionais de } \\
\text { Saúde Mental/ Equipes Multidisciplinares }\end{array}$ & $\mathrm{AE}, \mathrm{AH}, \mathrm{AJ}, \mathrm{AK}, \mathrm{BP}, \mathrm{BV}, \mathrm{CE}, \mathrm{DM}$ \\
\hline \multirow[t]{3}{*}{$\begin{array}{l}\text { Formadores/ Capacitadores/ } \\
\text { (Psico)Educativos }\end{array}$} & $\begin{array}{l}\text { Ações de Promoção à Saúde e Prevenção da } \\
\text { Doença COVID-19, Promover medidas de } \\
\text { proteção, segurança e necessidades básicas }\end{array}$ & $\mathrm{AB}, \mathrm{AC}, \mathrm{AI}, \mathrm{CO}, \mathrm{DN}$ \\
\hline & Educação em Saúde & $\mathrm{AR}, \mathrm{CM}$ \\
\hline & $\begin{array}{c}\text { Intervenções de mudanças de } \\
\text { comportamento, Dinâmica Cooperativa de } \\
\text { Treinamento, Ações Psicoeducativas, } \\
\text { Psicoeducação }\end{array}$ & $\begin{array}{c}\text { AD,AO,AQ,CA,CG,CK,EC,CY,DJ, } \\
\text { DM,ED,EN,FD }\end{array}$ \\
\hline \multirow[t]{2}{*}{ Serviço (ambiente laboral) } & $\begin{array}{l}\text { Ter ambiente adequado de trabalho, com } \\
\text { Equipamentos de Proteção Individual (EPI) e } \\
\text { assegurar as medidas de prevenção da doença }\end{array}$ & $\mathrm{AC}, \mathrm{AO}, \mathrm{AS}, \mathrm{AZ}, \mathrm{BF}, \mathrm{BJ}, \mathrm{EC}, \mathrm{EH}, \mathrm{FB}, \mathrm{FF}$ \\
\hline & $\begin{array}{c}\text { Medidas Organizativas do Serviço (mudança } \\
\text { de escala de profissionais e pacientes), } \\
\text { Assegurar continuidade de tratamento } \\
\text { farmacológico, Facilitar comunicações } \\
\text { Oficiais }\end{array}$ & BO,CE,CK,DL \\
\hline $\begin{array}{l}\text { Cuidado de Si (autocuidado } \\
\text { e autoconhecimento) }\end{array}$ & $\begin{array}{l}\text { Práticas de Relaxamento; Grupos de } \\
\text { Relaxamento; Atividades como caminhada, } \\
\text { canto; Ioga; Meditação; Mindfulness; } \\
\text { Técnica de Relaxamento Muscular } \\
\text { Progressivo; Meditação Guiada. }\end{array}$ & $\begin{array}{l}\text { AQ,AS,BO,CA,CE,CH,CN,DP,EE, } \\
\text { EN,CU,CX,FF,DI,EY,DO,EI,FG }\end{array}$ \\
\hline Puramente Emocionais & $\begin{array}{l}\text { Expor e conversar sobre preocupações e } \\
\text { necessidades; Classificar, validar e conversar } \\
\text { sobre emoções no trabalho; } \\
\text { Conversar sobre experiências e sentimentos; } \\
\text { Validação das emoções através de rodas de } \\
\text { conversa; Abordar sobre o impacto } \\
\text { psicológico e medidas de enfrentamento; } \\
\text { Expressar necessidades e sentimentos; } \\
\text { Compartilhar desafios e angústias; Ajudar a } \\
\text { entender e a identificar emoções; Informar } \\
\text { sentimentos de medo, ansiedade e tristeza }\end{array}$ & AY,BP,CE,EK,CM,CN,DI,DJ,FA,DS \\
\hline
\end{tabular}

Fonte: Autores (2021). 
Quadro 2 - Principais aspectos relacionados ao manejo das emoções pela sociedade na pandemia do Novo Coronavírus, 2021.

\begin{tabular}{|c|c|c|}
\hline Aspectos relacionados a(s)/ao(s) & Manejos & Referências \\
\hline $\begin{array}{l}\text { Fortalecimento e manutenção de } \\
\text { vínculos familiares e sociais }\end{array}$ & $\begin{array}{l}\text { Fortalecer vínculos; Dedicar-se a filhos e } \\
\text { cônjuge; Conversar com amigos e familiares } \\
\text { por videochamada; Apoio Social; Apoio de } \\
\text { familiares e amigos; Manter contato com } \\
\text { outras crianças; Fortalecer redes de apoio; } \\
\text { Fortalecer vínculo familiar e social; Manter } \\
\text { contato social. }\end{array}$ & $\begin{array}{c}\text { AN,AT,AU,AX,BW,DE,DS } \\
\text { DF,DX,BL,CF,CH,BM,BY,DN,DU,CC,CR } \\
\text {,EU,CY,EA, DA,EF,EH,ET,FE,FD. }\end{array}$ \\
\hline Atividades Físicas & $\begin{array}{l}\text { Rotina de Exercícios; Atividades Física } \\
\text { regularmente; Alongamento }\end{array}$ & $\begin{array}{c}\text { AF,AN,AT,AX,BC,BK,CH, } \\
\text { CK,CO,CP,CT,DT,DU,EB, } \\
\text { EF,EH,EP,EQ,ES,EM,EU, } \\
\text { FE,FB,FD,FF,FH }\end{array}$ \\
\hline $\begin{array}{l}\text { Limites de acesso a Informações sobre o } \\
\text { Novo Coronavírus }\end{array}$ & $\begin{array}{l}\text { Controlar e limitar o acesso a informação; } \\
\text { Acessar informações por meios confiáveis; } \\
\text { Monitorar e controlar informações da } \\
\text { pandemia; Acompanhar as últimas notícias, } \\
\text { incluindo progressos; Fortalecer meios de } \\
\text { comunicação; Evitar excesso de informações } \\
\text { e averiguar informações falsas. }\end{array}$ & $\begin{array}{l}\text { AF,AH,AN,AG,CK,CO,CU } \\
\text { DD,EA,FE,DS,EQ,AL,AO, } \\
\text { CA,CH,CY,DF,ES,FH }\end{array}$ \\
\hline $\begin{array}{l}\text { Atividades de } \\
\text { Lazer/Prazerosas }\end{array}$ & $\begin{array}{l}\text { Atividades de Distração; Atividades de } \\
\text { Lazer; Atividades Prazerosas }\end{array}$ & $\begin{array}{c}\text { AX,BC,BW,CO,CR,DE,DF } \\
\text { DS,DV,EQ,ER,FE,EF,FA, FC }\end{array}$ \\
\hline \multirow[t]{3}{*}{$\begin{array}{l}\text { Práticas Integrativas e meios culturais } \\
\text { de enfrentamento à crise }\end{array}$} & $\begin{array}{l}\text { Musicoterapia; Música como instrumento de } \\
\text { alto potencial para regulação emocional }\end{array}$ & AX,EJ,EX \\
\hline & $\begin{array}{c}\text { Relaxamento; Técnicas de Relaxamento; } \\
\text { Yoga }\end{array}$ & AT,AV,CH,CY,CT,DF,EH,FC \\
\hline & $\begin{array}{c}\text { Desenvolver habilidades para lidar com o } \\
\text { estresse; Contação de Histórias; Cultivar } \\
\text { Hobbies; Arteterapia; Pinturas; e, escrita de } \\
\text { Poemas }\end{array}$ & $\mathrm{AK}, \mathrm{CJ}, \mathrm{BA}, \mathrm{CI}$ \\
\hline Organização de Rotina Diária & $\begin{array}{l}\text { Rotina Familiar; Desenvolver atividades } \\
\text { ocupacionais (rotina doméstica); Estipular } \\
\text { horários de despertar e dormir. }\end{array}$ & $\begin{array}{c}\text { AT,AX,BA,BC,BW,CH,CK, } \\
\text { CO,CY,DF,ES,CC,DV,EF, } \\
\text { ER,FH,CV,ER,EU,FA }\end{array}$ \\
\hline
\end{tabular}

Fonte: Autores (2021).

No que tange aos aspectos que possibilitam autoconhecimento a partir da pandemia da COVID-19, 14 artigos (10\%) trouxeram as características e os manejos que devem ser observados a fim de se autoconhecer, que estão descritos no Quadro 3. 
Quadro 3 - Aspectos relacionados a manejos que possibilitam o autoconhecimento a partir das questões vivenciadas na pandemia do Novo Coronavírus, 2021.

\begin{tabular}{|c|c|c|}
\hline Aspectos relacionados & Manejos para construção do autoconhecimento & Referência(s) \\
\hline \multirow{3}{*}{$\begin{array}{l}\text { Modos de lidar consigo } \\
\text { mesmo subjetivamente } \\
\text { (autoconscientização) }\end{array}$} & compreender os sentimentos & $\mathrm{CR}$ \\
\hline & ter momentos de autorreflexão & $\mathrm{DF}$ \\
\hline & $\begin{array}{l}\text { Comportamentos de autoconfiança. controle do medo e } \\
\text { busca de ajuda }\end{array}$ & FB \\
\hline \multirow{2}{*}{$\begin{array}{l}\text { Modos de lidar consigo } \\
\text { mesmo de forma geral }\end{array}$} & hábitos de autocuidado & $\mathrm{BP}$ \\
\hline & entender que as emoções são respostas normais. & $\mathrm{CO}$ \\
\hline \multirow{3}{*}{$\begin{array}{l}\text { Traços de inteligência } \\
\text { emocional }\end{array}$} & ressignificar o momento pandêmico. & $\mathrm{CR}$ \\
\hline & adotar uma posição positiva & DD \\
\hline & $\begin{array}{l}\text { ser resiliente; resiliência; Ser resiliente; resiliência } \\
\text { como posição positiva }\end{array}$ & $\mathrm{AZ} ; \mathrm{BP} ; \mathrm{CF} ; \mathrm{DD} ; \mathrm{EY}$ \\
\hline \multirow{2}{*}{$\begin{array}{l}\text { Traços de Educação } \\
\text { Emocional }\end{array}$} & desenvolvendo habilidades de mindfulness & ED \\
\hline & controlar suas emoções diante as pessoas & $\mathrm{EZ}$ \\
\hline \multirow{2}{*}{$\begin{array}{l}\text { Meios externos que auxiliam } \\
\text { no desenvolvimento do } \\
\text { autoconhecimento }\end{array}$} & $\begin{array}{l}\text { Terapia de aceitação e compromisso, e terapia } \\
\text { comportamental dialética }\end{array}$ & ED \\
\hline & $\begin{array}{l}\text { Curso focado na atenção plena para } \\
\text { desenvolver habilidades socioemocionais, de bem-estar } \\
\text { e autoconsciência. }\end{array}$ & $\mathrm{EO}$ \\
\hline \multirow[t]{3}{*}{ Habilidades Socioemocionais } & $\begin{array}{l}\text { comunicação, empatia e pensamentos não ruminativos } \\
\text { são decisivos para facilitar tomadas de decisão. }\end{array}$ & $\mathrm{EY}$ \\
\hline & $\begin{array}{l}\text { Ter habilidades socioemocionais; saber expor seus } \\
\text { sentimentos }\end{array}$ & $\mathrm{EZ}$ \\
\hline & Reconhecer a vulnerabilidade da condição humana & $\mathrm{FC}$ \\
\hline
\end{tabular}

Fonte: Autores (2021).

\section{Discussão}

O estudo evidencia o crescimento expressivo da preocupação dos pesquisadores e profissionais de saúde mental acerca das demandas referentes às emoções e suas medidas de enfrentamentos relatadas por profissionais da saúde e também pela população em geral.

A partir dos resultados desta revisão é possível delimitar as principais emoções vivenciadas, assim como os principais manejos utilizados pelos profissionais e a sociedade em geral, a fim de enfrentar o período pandêmico da melhor maneira. Para além disso, a partir do estudo também destacam-se os aspectos que possibilitam desenvolver o autoconhecimento e inteligência emocional.

\section{Emoções à flor da pele na pandemia da COVID-19}

As emoções possuem a qualidade de ligar o externo com interno, estando no centro da experiência humana interna e social. As emoções permitem nossa adaptação, posto que são baseadas na relação entre eventos e nossas intenções e interesses. Desse modo, permitem que nos adaptamos às situações em que nos encontramos (Casassus, 2009).

O desencadeamento de emoções é um processo natural que ocorre diante de situações vivenciadas. No atual contexto mundial decorrente da pandemia da COVID-19, a percepção, principalmente de emoções tidas como negativas socialmente, como medo, raiva e tristeza, é um reflexo do momento difícil e incerto com o qual toda a população está lidando. Isso se justifica 
uma vez que o ser emocional existe num contexto social que afeta seus processos emocionais e condiciona as maneiras de agir diante das situações (Casassus, 2009).

A experiência emocional é de fato um fenômeno múltiplo, sendo resultado de vários eventos, que variam de pessoa para pessoa. Em uma situação simplificada a emoção se expressa por um ato motor, de natureza motivacional, que é desencadeado por sensações que são provocadas através de estímulos sensoriais do meio onde se encontra o indivíduo. No entanto, a emoção tem a possibilidade de incluir uma gama de pensamentos e planos sobre evento já ocorrido, que está ocorrendo ou que vai ocorrer e com isso, expressa-se sob expressões faciais características (Fonseca, 2013).

As expressões faciais características de cada emoção são universais, sendo o diferencial entre cada cultura as regras de exibição, isto é, o controle. Essas regras podem ditar a diminuição, o exagero ou o fingimento da expressão que sentimos. A tristeza e angústia nas expressões faciais e vocais pedem a ajuda de outros. Por isso, o apoio social, a atenção dos amigos e da família curam (Ekman, 2011). Tendo isso em consideração, ressalta-se a importância de se desenvolver a inteligência emocional, buscando conhecer suas próprias emoções mas também, sendo essencial, reconhecer as emoções das pessoas que te cercam a fim de poder auxiliá-las a lidar com a situação.

Os resultados encontrados nos estudos, tanto os que têm como foco profissionais da saúde, tanto os com a população em geral, apresentaram alta prevalência de emoções e características de emoções, como raiva, tristeza, angústia, depressão, ansiedade, estresse e preocupação, que são comumente reações a situações compreendidas pela sociedade de forma negativa (Ćosić, et al 2020 \& Mo, et al 2020). Essa atribuição de julgamentos entre emoções positivas/boas e negativas/ruins traz prejuízos em como expressar e manifestar essas emoções, uma vez que a tendência padrão é de reprimir as emoções negativas, dificultando não só o reconhecimento da emoção ou do sentimento que está sendo experienciado, mas também impactando em possíveis problemas psicossomáticos futuros, como dores crônicas.

As características de emoções, também chamadas de emoções secundárias, são estados emocionais derivados que se assemelham entre si, mas que apresentam diferentes nuances, podendo variar quanto a duração, objeto ou qualidades vivenciadas mais sutis (Casassus, 2009). Por apresentarem diferenças sobre aspectos como a intensidade e duração, além de serem respostas emocionais secundárias podem se apresentar também com estados de ânimo. Estes tratam-se de períodos mais extensos que as emoções e, normalmente, são mais comuns de serem identificados por quem os experimenta. Podem também mediar a forma como enxergamos o mundo ao redor, por exemplo quando estamos deprimidos, a tendência é adotar uma visão pessimista e mais melancólica das coisas ao redor, além de servir de base para disparar emoções.

Logo, diante de um cenário de pandemia, que impõe medidas de isolamento social e lockdown em alguns países, somado as notícias alarmantes diárias, as manifestações emocionais se tornam tão frequentes que podem se tornar prejudiciais, podendo evoluir para distúrbios de emoções, que são estados tóxicos que levam a patologias.

A nossa bagagem emocional, ou nossas reservas, são importantes para podermos lidar com as situações que demandam o disparar de nossas emoções, ou seja, quanto mais soubermos sobre as emoções que dispomos, mais teremos conhecimento daquelas que serão disparadas quando emergirem (Fonseca, 2013). A partir disso, ressalta-se a importância de aprender e ser capaz de identificar e nomear as emoções que estão emergindo diante das situações vividas, assim como buscar identificar os manejos que são eficazes e benéficos para cada indivíduo.

\section{O manejo das emoções frente ao novo normal}

Manejos das emoções pelos profissionais da saúde

Os aspectos relacionados aos serviços de saúde/ ambiente laboral apresentam-se como uma das principais medidas de enfrentamento utilizadas pelos trabalhadores da saúde, uma vez que atuam de forma a minimizar o risco de se infectar pelo novo coronavírus. A disponibilidade de equipamentos de proteção individual, assegurar a atuação de acordo com as medidas de 
prevenção, assim como garantir necessidades básicas, como descanso, são medidas eficazes na diminuição do medo, ansiedade e preocupação. A necessidade de promover segurança dos profissionais da linha de frente do enfrentamento, garantindo tanto recursos físicos como recursos humanos adequados, são observadas em protocolos de respostas a epidemias anteriores (O'Sullivan, et al., 2007).

No caso da COVID-19, a eficácia do EPI está relacionada ao fornecimento de equipamentos com a proteção suficiente para o SARS-CoV-2 e o treinamento adequado das equipes de trabalhadores para o uso correto e consistente (Centers for Disease Control and Prevention apud Gallasch, et al., 2020). Essa afirmativa vai de encontro a outro importante manejo dos profissionais que diz respeito a aspectos educativos. A necessidade de organizar treinamentos e fortalecer a capacitação da equipe, como também estimular a participação e comunicação entre a equipe são essenciais para oferecer segurança ao profissional e ao paciente que recebe os cuidados (Mo et al., 2020).

O cenário pandêmico, além de se apresentar como um desafio por ser uma situação com a qual alguns profissionais nunca vivenciaram, constitui um período de constantes incertezas e falta de informações sobre o vírus SARS-CoV-2. Somado a isto, o contexto de ampla disseminação de notícias falsas dificulta e se caracteriza como mais um estressor. Diante disso, ressaltase não só a promoção da troca de informação e treinamento adequado entre as equipes, mas a necessidade de ter acesso a canais oficiais e confiáveis sobre a COVID-19. Mediante estratégias que facilitem a comunicação das informações para esses profissionais, por meios oficiais e com ampla divulgação, minimizando e esclarecendo notícias falsas, espera-se que se sintam mais seguros, contribuindo para a prevenção de sintomas psíquicos como medo e ansiedade (Saidel, et al., 2020.)

Os manejos referentes aos serviços também devem incluir, além das proteções físicas, a oferta de vacinação e/ou terapias antivirais, e deve se estender a identificação e notificação de casos suficientes (O'Sullivan, et al., 2007). A partir disso, é importante destacar que o medo, preocupações e angústias, mesmo que sejam minimizados seguindo os protocolos de paramentação e desparamentação dos materiais de forma correta, ainda estão presentes cotidianamente na rotina desses trabalhadores, uma vez que ainda não existe um tratamento mundial divulgado e eficaz para a COVID-19 e ações de vacinação e rastreamento dos casos da doença ainda são realizados de forma ineficaz para conter a pandemia.

Além disso, outra categoria encontrada foram os aspectos referentes ao autocuidado e autoconhecimento. Atividades que proporcionam qualidade de vida e bem-estar psicológico devem ser preconizadas com intuito de amenizar o estresse mental. A realização de atividades físicas e de práticas integrativas e complementares em saúde são estratégias que têm sido buscadas de forma crescente como forma maneiras de cuidar de si e prestar mais atenção a si. As práticas integrativas e complementares, como o relaxamento, representa um estado de expansão do organismo em contraste com o de tensão, que é o estado de contração, estando inteiramente relacionado à respiração livre e desbloqueada para ser efetivado com plenitude. Da mesma forma que a meditação é um meio de silenciamento do barulho do mundo externo e, consequentemente, o contato com o mundo interior, mediante o relaxamento corporal. (Lowen apud Carneiro, et al., 2020.). Logo, a partir de momentos de meditação e relaxamento, o profissional da saúde tem a possibilidade de focar sua atenção em suas emoções e sentimentos.

Tendo isso em vista, essas práticas possuem um grande impacto no que tange ao desenvolvimento da inteligência emocional, uma vez que ao se permitir esvaziar os pensamentos e entrar num estado de relaxamento, é possível que emoções ou características de emoções que ainda não foram identificadas fiquem em evidência. Conhecer as emoções e identificar quando se está experienciando determinada emoção é relevante não só para buscar a melhor estratégia para lidar com a situação, mas também para se autoconhecer melhor e ser capaz de identificar de que forma essas se manifestam.

Manejos das emoções pela sociedade

No que tange aos manejos das emoções pela sociedade, cabe destacar que assim como existe preocupação dos profissionais da saúde sobre as informações da pandemia, a população em geral também busca constantemente novas informações sobre a COVID-19. Por isso, um aspecto relevante para manejar as emoções é limitar o acesso às informações sobre 
o coronavírus. É necessário que a sociedade faça um uso consciente da informação, buscando limitar o período de tempo que fica exposto apenas a notícias ruins sobre a pandemia, assim como buscar notícias boas e informações sobre pessoas que se recuperaram. (Fiorillo \& Gorwood, 2020; Li, et al., 2020.).

Outro cuidado que se deve ter, em relação às notícias, é checar a fonte e se são notícias confiáveis. Nessa busca por informações, algumas pessoas acabam confiando em todo tipo de notícia que encontram em suas redes sociais, sem procurar saber a veracidade da informação. Assim, são disseminadas as Fake News de conteúdos diversos, como receitas milagrosas, falsas notícias sobre a origem da doença, profecias e meios de prevenção que não funcionam. Esse tipo de conteúdo impressiona as pessoas que se encontram em um momento difícil, confuso e, por vezes, com um cenário de medo. Tais informações não verídicas acabam prejudicando ainda mais o cotidiano e a saúde das pessoas, além de provocar o caos e o desespero (De Sousa Júnior, et al, 2020). Nesse sentido, uma prática que deveria auxiliar no enfrentamento da pandemia, se realizada de forma excessiva, pode acabar gerando mais estressores e dificuldades.

Além disso, a população também pode realizar atividades prazerosas, como atividades de lazer, práticas integrativas e atividades físicas, a fim de buscar momentos de descontração e relaxamento que promovam tanto bem estar físico como mental. A prática de atividades físicas são atividades que apresentam fortalecimento do sistema imunológico, sendo positivo para diminuir a incidência de infecções virais, e atuam também na regulação emocional, uma vez que apresentam diminuição de estresse e ansiedade, e promovem ampliação de emoções positivas(Raiol, et al.,2020). Tais resultados encontrados no estudo vão de encontro ao exposto em estudos anteriores sobre a relação entre prática regular de exercícios e saúde mental, uma vez que a prática do exercício físico pode funcionar de forma preventiva em intervenções terapêuticas multimodais, fomentando a diminuição de sintomatologia depressiva e ansiosa e a melhoria de funções cognitivas. (Knochel et al., 2012 apud Odília \&Simões, 2017).

Uma forma interessante de relacionar a prática de atividades físicas com atividades de lazer é a realização de Exergames. Eles são um tipo de jogo de videogame não sedentário, o qual requer esforço físico do participante para ser jogado. Os exergames têm se apresentado como uma possibilidade divertida e eficaz de promover atividade física em casa. Usualmente, os exergames são jogados por meio de plataformas comerciais de jogos eletrônicos, o que pode facilitar o acesso das pessoas para terem esse recurso tecnológico em suas residências (Rocha, et al, 2020). A utilização de exergames como maneira de se divertir e ao mesmo tempo se exercitar é um recurso interessante de ser utilizado por idosos, que são grupos que apresentam vulnerabilidades de saúde física e mental e que foram amplamente afetados pelas medidas de isolamento, uma vez que se caracterizam como grupo de risco da COVID-19, mas também por crianças e jovens, que também estão passando por um período difícil, logo, o tédio e a solidão podem ser minimizados praticando essas atividades. Por serem realizadas por plataformas onlines, os exergames se caracterizam como uma prática que pode ser realizada tanto de forma individual, como em grupos, podendo ser jogado com amigos e familiares, favorecendo também a socialização.

A utilização de plataformas onlines tem sido o principal recurso utilizado no período da pandemia com intuito de encurtar o distanciamento e promover a interação entre amigos e familiares. Fortalecer e manter os vínculos familiares e sociais é essencial nesse período, sentimentos de solidão, angústia e depressão podem ser minimizados em momentos de conversas e troca entre amigos ou familiares.

Poder contar com o apoio social, principalmente em momentos conturbados é importante uma vez que as pessoas têm a capacidade de serem envolvidas por contágios emocionais. Transmitimos e captamos modos uns dos outros, algo como uma economia subterrânea da psique, em que alguns encontros são tóxicos, outros, revigorantes(Goleman, 2011). Tendo isso em vista, é possível que em momentos de ansiedade ou angústia, ao buscar o apoio de amigos ou familiares, compartilhar suas vivências possibilitem emergir emoções e estados de espíritos positivos. 


\section{O autoconhecimento como caminho para a construção da Inteligência Emocional - atalhos e desvios.}

O autoconhecimento é o princípio base para se desenvolver as aptidões da inteligência emocional. Estar atento, desenvolver a consciência sobre o que ocorre dentro de si é a característica inicial para buscar entender os padrões e reações, tanto do corpo quanto da mente, diante das situações vividas.

Realizar práticas que viabilizem modos de lidar consigo de modo geral ou subjetivamente foram encorajados e aprofundados nesse período de pandemia. Ter capacidade de estar aberto ao mundo emocional assim como de estar atento são algumas das competências necessárias para agir no mundo emocional. (Casassus, 2009). A fim de desenvolver essas capacidades é necessário que ações, como: compreender os sentimentos, ter momentos de autorreflexão, práticas de mindfulness e hábitos de autocuidado sejam realizadas e tidas como importantes. É preciso atribuir importância ao mundo emocional, para desenvolver a consciência necessária e estar atento a si. Pois, somente ao se dar conta do que está acontecendo, ou das emoções que se está sentindo, é possível avaliar a melhor resposta e buscar atitudes que irão proporcionar bem-estar.

Uma característica importante a ser discutida é a possibilidade de transformação a partir da autoconsciência. Ao tomarmos consciência do nosso corpo, de seus vínculos com as emoções, e com as narrativas que construímos sobre elas, é possível ver o que não era visto antes(Casassus, 2009). Por isso, é possível que após perceber padrões de comportamento e o impacto que algumas situações causam, seja possível compreender e lidar com a situação de outra forma.

As emoções são energias neutras, e respostas normais às situações da vida, logo a maneira como lidamos com ela é um resultado de um processo de aprendizagem, e atribuição de pensamentos e subjetividade, iniciado na infância(Casassus,2009). O desenvolvimento da inteligência emocional, começando pelo reconhecimento das emoções e como essas afetam o cotidiano, surge como uma possibilidade de aprender e explorar novas maneiras de lidar com essas situações. Isso ocorre uma vez que o cérebro tem a capacidade de se remodelar a partir de experiências repetidas, sendo essa característica chamada de neuroplasticidade. Logo, no contexto da pandemia, no qual as mesmas emoções e sentimentos têm sido disparados de forma recorrente, a educação emocional é um importante caminho para buscar o bem-estar e manter uma boa saúde mental.

A educação do ser emocional não consiste em apagar a memória do evento, mas em separar a minha reação da ideia desse. A capacidade de regulação emocional, é um processo interno que acontece em três momentos: a tomada de consciência; o reconhecimento; e a domesticação da emoção (Casassus, 2009). Por meio de atitudes que proporcionem expor seus sentimentos, e perceber estes de forma consciente e não apenas uma reação automática, assim como desenvolver habilidades para controlar o medo e raiva.

Outro instrumento de domesticação das emoções é o pensamento (Casassus, 2009). As emoções emergem no corpo na forma de energias, e é apenas quando atribuímos pensamentos, sejam positivos ou negativos a situação, que a emoção também adquire essa característica. Tendo isso em vista, é possível que ao ressignificar o momento e atribuir outros pensamentos à situação, a forma de lidar com a experiência seja alterada. Isso não significa reprimir as emoções, mas sim buscar prestar atenção na emoção que emerge, identificá-la e posteriormente questionar se o significado que está sendo atribuído é um pensamento consciente ou uma expressão do inconsciente emocional.

Nos momentos em que emoções com forte intensidade surgem são caracterizadas sequestros neurais, isto é, nesses momentos o cérebro límbico dispara informações de forma mais rápida para outras áreas do cérebro, antes que o neocórtex, o cérebro pensante, seja capaz de mediar a situação (Goleman, 2011). Isto posto, é evidente que o desenvolvimento da inteligência emocional constitui uma maneira eficaz de buscar o equilíbrio emocional, mas se trata de um caminho que precisa ser exercitado e aprimorado a cada dia. Buscar momentos para voltar a atenção para si visando o autoconhecimento proporciona, além do reconhecimento próprio, caminhos para lidar com a vida e as pessoas ao redor de forma mais empática e equilibrada. 


\section{Limitações do estudo}

O estudo apresenta algumas limitações. Por se tratar de uma revisão integrativa de literatura, não foram acessadas todas as bases disponíveis online para realizar a busca dos artigos. Além disso, o período de busca dos artigos foi limitado apenas a artigos publicados e disponíveis online no ano de 2020.

\section{Conclusão}

O estudo demonstrou que o período da pandemia da COVID-19, suscitou uma série de emoções, que podem potencializar transtornos mentais, gerando aumento do fluxo de usuários na Rede pública de Atenção Psicossocial e serviços psiquiátricos privados. A exposição constante a gatilhos emocionais associados a emoções frequentes e intensas podem ser desencadeantes de estados mentais patológicos, sendo necessário buscar, além das medidas de enfrentamento cotidianas, assistência especializada de profissionais de saúde mental.

Além disso, diversas formas de lidar com as emoções foram exemplificadas nos diferentes manejos, evidenciando a importância de se conhecer e reconhecer as emoções que se manifestam e identificar a melhor forma de manejar essas emoções e sentimentos. O período de distanciamento social imposto pela pandemia da COVID-19, além de aflorar muitas emoções e sentimentos concomitantemente, também se apresenta como um momento que permite maior maior autoconscientização das emoções vivenciadas. Por isso, se apresenta como cenário de desenvolvimento da inteligência emocional.

Por fim, sugere-se que a partir dos achados, outros estudos possam aprofundar a temática in voga, uma vez que podem auxiliar no aprofundamento do autoconhecimento das pessoas, ferramenta essencial ao desenvolvimento da inteligência e educação emocional, por exemplo. Tais estratégias vêm mostrando resultados relevantes no campo da promoção e manutenção da saúde mental, uma vez que contempla legitimamente as demandas daqueles que se dispõem a iniciarem e porem em prática este exercício.

\section{Fomento}

O presente trabalho foi realizado com apoio financeiro do CNPq/PIBIC através da concessão de bolsa do Programa Institucional de Bolsas de Iniciação Científica - PIBIC-UFRJ.

\section{Referências}

Bardin, L. (2011). Análise de conteúdo. Edições 70.

Broche Pérez, Y., Fernández Castillo, E. \& Reyes Luzardo, D. A. (2020). Consecuencias psicológicas de la cuarentena y el aislamiento social durante la pandemia de COVID-19. Revista Cubana de Salud Pública, 46(supl.1): e2488. http://www.revsaludpublica.sld.cu/index.php/spu/article/view/2488.

Carneiro, J., Caribé, C. \& Rego, G. (2021). PICS em saúde mental: Oficinas de relaxamento e meditação. REVISE - Revista Integrativa em Inovações Tecnológicas nas Ciências da Saúde, 5, 157-175. https://www3.ufrb.edu.br/seer/index.php/revise/article/view/1384.

Casassus, J. (2009).Fundamentos da educação emocional. UNESCO, Liber Livro Editora.

Ćosić, K, et al.(2020). Impact of Human Disasters and COVID-19 Pandemic on Mental Health: Potential of Digital Psychiatry. Psychiatria Danubina, 32 (1), 25-31. https://doi.org/10.24869/psyd.2020.25.

De Sousa Júnior, J. H, et al. (2020). Da Desinformação ao Caos: uma análise das Fake News frente à pandemia do Coronavírus (COVID-19) no Brasil. Cadernos de Prospecção, 13 (2), 331. http://dx.doi.org/10.9771/cp.v13i2.COVID-19.35978.

Ekman, P. (2011). A linguagem das emoções. Ed. Lua de Papel.

Fiorillo, A. \& Gorwood, P. (2020). The consequences of the COVID-19 pandemic on mental health and implications for clinical practice. European Psychiatry, Cambridge, 63(1), e32.https://doi.org/10.1192/j.eurpsy.2020.35.

Fonseca, P. I. M. N. (2013). Emoções vivenciadas pela equipe multiprofissional de uma central de transplantes na entrevista familiar. Dissertação (Mestrado). Escola de Enfermagem Aurora de Afonso Costa (EEAAC)/UFF.

Gallasch, C. H., et al. (2020). Prevenção relacionada à exposição ocupacional do profissional de saúde no cenário de COVID-19. Revista Enfermagem UERJ, 
28, 49596. https://doi.org/10.12957/reuerj.2020.49596.

Goleman, D. (2011). Inteligência Emocional. Ed.Objetiva.

Jakovljevic, M. COVID-19 Crisis as a Collective Hero's Journey to Better Public and Global Mental Health. Psychiatria Danubina, 32 (1), 3-5. https://doi.org/10.24869/psyd.2020.3.

Li, S., et al. (2020). The Impact of COVID-19 Epidemic Declaration on Psychological Consequences: A Study on Active Weibo Users. Int. J. Environ. Res. Public Health, $\quad$ Switzerland, 17(6),20-32. http://content.ebscohost.com/ContentServer.asp?T=P\&P=AN\&K=32204411\&S=R\&D=md c\&EbscoContent=dGJyMNHr7ESep7U4y9f3OLCmsEieqK5Ssqa4S66WxWXS\&ContentCustomer=dGJyMPGqsUuurrZQuePfgeyx44Dt6fIA.

Magalhães, G. \& Cardoso, L.C.B. (2020). Efeitos Econômicos e Distributivos da pandemia de Coronavirus no Brasil. Revista de Economia e Agronegócio, 18(1), 1-12. file:///C:/Users/Aluno/Downloads/10605-Texto\%20do\%20artigo-46708-1-10-20200624.pdf.

Ministério da Saúde. (2021). Painel Coronavírus. https://covid.saude.gov.br/

Mo, Y., et al. (2020). Work stress among Chinese nurses to support Wuhan for fighting against the COVID-19 epidemic. Journal of nursing management. https://onlinelibrary.wiley.com/doi/abs/10.1111/jonm.13014.

Odília, A. M. \& Simões Dias, I. (2017). Exercício Físico, Saúde Mental E Qualidade De Vida Na ESECS/IPL.L. Psicologia, Saúde e Doenças, 18(2),512-526. http://dx.doi.org/10.15309/17psd180219.

Organização Pan-Americana da Saúde. (2021) Folha informativa COVID-19 - Escritório da OPAS e da OMS no Brasil. https://www.paho.org/pt/covid19

O'Sullivan, T. L. et al. (2007). Are We Ready? Evidence of Support Mechanisms for Canadian Health Care Workers in Multi-jurisdictional Emergency Planning. Canadian journal of public health, 98(5),358-363. http://content.ebscohost.com/ContentServer.asp?T=P\&P=AN\&K=17985675\&S=R\& $\mathrm{D}=$ mdc\&EbscoContent=dGJyMNHr7ESep7U4y9f3OLCmsEieqK9Srq\%2B4SbKWxWXS\&ContentCustomer=dGJyMPGqsUuurrZQuePfgeyx44Dt6fIA.

Raiol, R. A. (2020). Praticar exercícios físicos é fundamental para a saúde física e mental durante a Pandemia da COVID-19. Brazilian Journal of health Review.,3(2),2804-2813. http://www.brazilianjournals.com/index.php/BJHR/article/view/8463/7298.

Ribeiro L. M., Vieira, T. de A. \& Naka K. S. (2020). Síndrome de burnout em profissionais de saúde antes e durante a pandemia da COVID-19. Revista Eletrônica Acervo Saúde, 12(11), e5021. https://doi.org/10.25248/reas.e5021.2020.

Rocha, S. V., et al. (2021). A pandemia de COVID-19 e a saúde mental de idosos: possibilidades de atividade física por meio dos Exergames. Rev Bras Ativ Fís Saúde, 25. https://rbafs.org.br/RBAFS/article/view/14424.

Saidel, M. G. B., et al. (2020). Intervenções em saúde mental para profissionais de saúde frente a pandemia de Coronavírus . Revista Enfermagem UERJ, 28, e49923. https://doi.org/10.12957/reuerj.2020.49923.

Souza, M. T., Silva, M. D. \& Carvalho, R.(2010). Revisão integrativa: o que é e como fazer. Einstein (São Paulo), 8(1),102-106. https://doi.org/10.1590/s167945082010 rw1134.

\section{Bibliografias Consultadas}

AA. Wang, C., et al. (2020).A longitudinal study on the mental health of general population during the COVID-19 epidemic in China. Brain, Behavior, and Immunity, Disponível em: https://www.sciencedirect.com/science/article/pii/S0889159120305110?via\%3Dihub.https://doi.org/10.1016/j.bbi.2020.04.028.

AB. Sun, N., et al. (2020).A qualitative study on the psychological experience of caregivers of COVID-19 patients. Am J Infect Control., 00 1-7.. https://doi.org/10.1016/j.ajic.2020.03.018.

AC. Albott, C. S., et al. (2020).Battle Buddies: Rapid Deployment of a Psychological Resilience Intervention for Healthcare Workers during the COVID-19 Pandemic. Anesthesia and analgesia, Publish Ahead of Print.doi:10.1213/ANE.0000000000004912.

AD. Jakovljevic, M. (2020). COVID-19 Crisis as a Collective Hero's Journey to Better Public and Global Mental Health. Psychiatria Danubina, 32(1),3-5. https://doi.org/10.24869/psyd.2020.3.

AE.Shuja,K. H., et al. (2020).COVID-19 Pandemic and Impending Global Mental Health Implications. psychiatria danubina, 32(1),32-35. https://doi.org/10.24869/psyd.2020.32.

AF. Huang, Y.; Zhao, N. (2020). Generalized anxiety disorder, depressive symptoms and sleep quality during COVID-19 outbreak in China: a web-based crosssectional survey. Psychiatry Research, 288. https://doi.org/10.1016/j.psychres.2020.112954

AG. Wang, C., et al.(2020). Immediate Psychological Responses and Associated Factors during the Initial Stage of the 2019 Coronavirus Disease (COVID-19) Epidemic among the General Population in China. Int. J. Environ. Res. Public Health, 17(5).Doi:10.3390/ijerph17051729

AH. Ćosić, K., et al. (2020).Impact of Human Disasters and COVID-19 Pandemic on Mental Health: Potential of Digital Psychiatry. Psychiatria Danubina, 32(1),25-31. https://doi.org/10.24869/psyd.2020.25.

AI. Zhang, Y.i \& Ma, Z. F. (2020). Impact of the COVID-19 Pandemic on Mental Health and Quality of Life among Local Residents in Liaoning Province, China: A Cross-Sectional Study. Int. J. Environ. Res. Public Health,17(7).doi:10.3390/ijerph17072381.

AJ. Kang, L., et al. (2020).Impact on mental health and perceptions of psychological care among medical and nursing staff in Wuhan during the 2019 novel coronavirus disease outbreak: A cross-sectional study. brain, behavior, and immunity.https://doi.org/10.1016/j.bbi.2020.03.028.

AK. Tan, W., et al. Is returning to work during the COVID-19 pandemic stressful? A study on immediate mental health status and psychoneuroimmunity 
prevention measures of Chinese workforce. Brain, Behavior, and Immunit. https://www.sciencedirect.com/science/article/pii/S0889159120306036?via\%3Dihub. https://doi.org/10.1016/j.bbi.2020.04.055.

AL. Gao, J., et al.(2020). Mental health problems and social media exposure during COVID-19 outbreak. PLoS ONE, 15(4).https://doi.org/ 10.1371/journal.pone.0231924.

AM. Lu, W., et al. Psychological status of medical workforce during the COVID-19 pandemic: A cross-sectional study. Psychiatry Research,. 288. https://doi.org/10.1016/j.psychres.2020.112936.

AN. Fiorillo, A. \& Gorwood, P. (2020). The consequences of the COVID-19 pandemic on mental health and implications for clinical practice. European Psychiatry,63(1),e32. https://doi.org/10.1192/j.eurpsy.2020.35.

AO. Li, S., et al. (2020). The Impact of COVID-19 Epidemic Declaration on Psychological Consequences: A Study on Active Weibo Users. Int. J. Environ. Res. Public Health,17(6),20-32. doi:10.3390/ijerph17062032.

AP. Cao, W., et al. (2020). The psychological impact of the COVID-19 epidemic on college students in China. Psychiatry Research, 287. https://doi.org/10.1016/j.psychres.2020.112934.

AQ. Mo, Y., et al. (2020). Work stress among Chinese nurses to support Wuhan for fighting against the COVID-19 epidemic. Journal of nursing management. doi: 10.1111/JONM.13014.

AR. Yuan, S., et al. (2020). Comparison of the Indicators of Psychological Stress in the Population of Hubei Province and Non-Endemic Provinces in China During Two Weeks During the Coronavirus Disease 2019 (COVID-19) Outbreak in February 2020. Medical science monitor,26. doi:10.12659/MSM.923767.

AS. Ripp, J., Peccoralo, L. \& Charney, D. (2020). Attending to the Emotional Well-Being of the Health Care Workforce in a New York City Health System During the COVID-19 Pandemic.Academic Medicine. Doi10.1097/ACM.0000000000003414.

AT. Kazerooni, A. R., et al. (2020).Peer mentoring for medical students during COVID-19 pandemic via a social media platform. Medical Education Adaptations: Really Good Stuff for Educational Transition during a Pandemic.https://doi.org/10.1111/medu.14206 .

AU. Cerbara, L., et al.(2020). A nation-wide survey on emotional and psychological impacts of COVID-19 social distancing. Eur Rev Med Pharmacol Sci, 24(12), 7155-7163. DOI: 10.26355/eurrev_202006_21711

AV. Parimala, S. \& Kanchibhotla, D. (2020).Association Between Yogic Breathing Practice With Perceived Impact of COVID-19: A Cross-Sectional Study From India. Asia Pacific Journal of Public Health. .doi:10.1177/1010539520975282

AW. George, C. E, et al. (2020).Challenges, experience and coping of health professionals in delivering healthcare in an urban slum in India during the first 40 days of COVID-19 crisis: a mixed method study. BMJ Open, 10(11). doi: 10.1136 / bmjopen-2020-042171

AX. Singh, N., et al. (2020). Concerns and coping strategies of persons under institutional quarantine during SARS-CoV-2 pandemic. Indian J Palliat Care, 26, 99-10.doi: 10.4103/IJPC.IJPC_176_20

AY. Stephens, E.H., Dearani J.A. \& Guleserian K.J. (2020). Courage, Fortitude, and Effective Leadership of Surgical Teams During COVID-19. World J Pediatr Congenit Heart Surg, 11(5),.675-679. doi: 10.1177/2150135120938330.

AZ. Vanhaecht, K., et al. (2020).COVID-19 is having a destructive impact on health-care workers' mental well-being. International Journal for Quality in Health Care. https://doi.org/10.1093/intqhc/mzaa158

BA. Zhang, Yan et al.(2020). Emotional "inflection point" in public health emergencies with the 2019 new coronavirus pneumonia (NCP) in China. Journal of Affective Disorders, v. 276, p. 797-803. https://doi.org/10.1016/j.jad.2020.07.097.

BB. Huang, L.., et al.(2020). Emotional responses and coping strategies in nurses and nursing students during COVID-19 outbreak: A comparative study. PloS one, v. 15,ed. 8, p.237-303, Aug. doi:10.1371/journal.pone.0237303

BC. Shanahan, L., et al.(2020). Emotional distress in young adults during the COVID-19 pandemic: evidence of risk and resilience from a longitudinal cohort study. Psychological medicine, Jun.. doi:10.1017/S003329172000241X

BD. Consolo, U., et al.(2020). Epidemiological Aspects and Psychological Reactions to COVID-19 of Dental Practitioners in the Northern Italy Districts of Modena and Reggio Emilia. International Journal of Environmental Research and Public Health, v. 17, ed. 10 3459, May. doi:10.3390/ijerph17103459

BE. Embregts, P.J.C.M., Tournier, T, Frielink, N.(2021). Experiences and needs of direct support staff working with people with intellectual disabilities during the COVID-19 pandemic: A thematic analysis. J Appl Res Intellect Disabil, v.34, p. 480-490, March .. https://doi.org/10.1111/jar.12812

BF. Galehdar, N., et al.(2020). Exploring nurses' experiences of psychological distress during care of patients with COVID-19: a qualitative study. BMC Psychiatry, V. 20, n.489, October . https://doi.org/10.1186/s12888-020-02898-1

BG. Sweeny, K., et al.(2020).Flow in the time of COVID-19: Findings from China. Plos One, v.15, ed.11, Nov. https://journals.plos.org/plosone/article?id=10.1371/journal.pone.0242043\#abstract0. https://doi.org/10.1371/journal.pone.0242043

BH. White, E.M., et al.(2021). Front-line Nursing Home Staff Experiences During the COVID-19 Pandemic. J Am Med Dir Assoc, v. 22, ed.1, p.199-203, Jan. doi: 10.1016/j.jamda.2020.11.022.

BI. Karasmanaki, E., Tsantopoulos, G.(2021). Impacts of social distancing during COVID-19 pandemic on the daily life of forestry students. Child Youth Serv Rev, v.120, Jan .doi:10.1016/j.childyouth.2020.105781.

BJ. Leng, M., et al.(2020). Mental distress and influencing factors in nurses caring for patients with COVID-19. Nurs Crit Care, online. https://doi.org/10.1111/nicc. 12528 
BK. Zhang, Yao., et al.(2020).Mental Health Problems during the COVID-19 Pandemics and the Mitigation Effects of Exercise: A Longitudinal Study of College Students in China. International Journal of Environmental Research and Public Health,v.17,ed.10, May . doi:10.3390/ijerph17103722

BL. Brooks, S.K., Weston, D., Greenberg, N.(2020). Psychological impact of infectious disease outbreaks on pregnant women: rapid evidence review. Public Health,v. 189, p.26-36 .doi:10.1016/j.puhe.2020.09.006

BM. Sun, N., et al. (2021).Qualitative study of the psychological experience of COVID-19 patients during hospitalization. J Affect Disord, v.278,p.15-22, Jan. doi: 10.1016/j.jad.2020.08.040.

BN. Giordano, F., et al.(2020). Receptive music therapy to reduce stress and improve wellbeing in Italian clinical staff involved in COVID-19 pandemic: A preliminary study. The Arts in Psychotherapy, v. 70. doi:10.1016/j.aip.2020.101688

BO. Iheduru-Anderson, K.(2021). Reflections on the lived experience of working with limited personal protective equipment during the COVID-19 crisis. Nurs Inq, v.28,ed.1. https://doi.org/10.1111/nin.12382

BP. Mellins, C.A., et al. (2020).Supporting the well-being of health care providers during the COVID-19 pandemic: The CopeColumbia response.General Hospital Psychiatry, v. 67, p.62-69. doi:10.1016/j.genhosppsych.2020.08.013

BQ. Ares, G., et al.(2021). The experience of social distancing for families with children and adolescents during the coronavirus (COVID-19) pandemic in Uruguay: Difficulties and opportunities. Child Youth Serv Rev, v.121, Feb..doi:10.1016/j.childyouth.2020.105906.

BR. Iglesias-Sánchez, P.P., et al.(2020). The Contagion of Sentiments during the COVID-19 Pandemic Crisis: The Case of Isolation in Spain. Int. J. Environ. Res. Public Health, v.17, ed. 16. https://doi.org/10.3390/ijerph17165918

BS. Li, Q., et al.(2020).Tracking and Analyzing Public Emotion Evolutions During COVID-19: A Case Study from the Event-Driven Perspective on Microblogs. Int J Environ Res Public Health,v.17,ed.18, Sep .doi: 10.3390/ijerph17186888. PMID: 32967163; PMCID: PMC7559419.

BT. Moroń, M. \& Biolik-Moroń, M.(2021). Trait emotional intelligence and emotional experiences during the COVID-19 pandemic outbreak in Poland: A daily diary study. Personality and Individual Differences, v. 168. doi:10.1016/j.paid.2020.110348

BU. Collado-Boira, E.J., et al.(2020). "The COVID-19 outbreak"-An empirical phenomenological study on perceptions and psychosocial considerations surrounding the immediate incorporation of final-year Spanish nursing and medical students into the health system. Nursing Education Today, v. 92. doi:10.1016/j.nedt.2020.104504

BV. Huarcaya-Victoria, J.(2020). Consideraciones sobre la salud mental en la pandemia de COVID-19. Rev Peruana de Medicina Experimental y Salud Pública, v.37, ed.2, p. 327-34. DOI: https://doi.org/10.17843/rpmesp.2020.372.5419

BW. Son, C., et al.(2020)..Effects of COVID-19 on College Students' Mental Health in the United States: Interview Survey Study. J Med Internet Rev, v.22, ed.9. DOI: $10.2196 / 21279$

BX. Giordani, R.C.F., et al.(2020). Fear of COVID-19 scale: Assessing fear of the coronavirus pandemic in Brazil. J Health Psychol, online. DOI: $10.1177 / 1359105320982035$.

BY. Gan, Y., et al.(2020). Immediate and delayed psychological effects of province-wide lockdown and personal quarantine during the COVID-19 outbreak in China. Psychological Medicine, p.1-12. doi:10.1017/S0033291720003116

BZ. Elsharkawy, N.B., Abdelaziz, E.M.(2020) Levels of fear and uncertainty regarding the spread of coronavirus disease (COVID-19) among university students. Perspect Psychiatr Care, online. https://doi.org/10.1111/ppc.12698

CA. Roy, A., et al.(2020). Mental health implications of COVID-19 pandemic and its response in India. International Journal of Social Psychiatry, online. doi:10.1177/0020764020950769

CB. Stuijfzand, S., et al.(2020).Psychological impact of an epidemic/pandemic on the mental health of healthcare professionals: a rapid review. BMC Public Health, v.20. https://doi.org/10.1186/s12889-020-09322-z

CC. Abdulah, D.M., Abdulla, B.M.O., \& Liamputtong P.(2020). Psychological response of children to home confinement during COVID-19: A qualitative artsbased research. International Journal of Social Psychiatry, online..doi:10.1177/0020764020972439

CD. Crowe,S., et al.(2020).The effect of COVID-19 pandemic on the mental health of Canadian critical care nurses providing patient care during the early phase pandemic: A mixed method study. Intensive \& Critical Care Nursing, online..https://doi.org/10.1016/j.iccn.2020.102999

CE. Muller, A.E., et al.(2020). The mental health impact of the COVID-19 pandemic on healthcare workers, and interventions to help them: A rapid systematic review. Psychiatry Rev, v.293. doi:10.1016/j.psychres.2020.113441.

CF. Serafini, G., et al.(2020). The psychological impact of COVID-19 on the mental health in the general population. QJM: An International Journal of Medicine, v.113, ed.8,p. 531-537. https://doi.org/10.1093/qjmed/hcaa201

CG. Ornell, F., et al.(2020). O impacto da pandemia de COVID-19 na saúde mental de profissionais de saúde. Cadernos de Saúde Pública , Rio de Janeiro, v. 36, n. 4, e00063520, 2020. http://dx.doi.org/10.1590/0102-311x00063520.

CH. Schmidt, B., et al.(2020). Impacts on Mental Health and Psychological Interventions related to the New Coronavirus Pandemic (COVID-19). Scientific Electronic Library Online. https://doi.org/10.1590/SciELOPreprints.58

CI. Medeiros, M.S., et al.(2020). A Arte como Estratégia de Coping em Tempos de Pandemia. Rev. Bras. Educ. Med., Brasília , v. 44, ed. 1, e130. https://doi.org/10.1590/1981-5271v44.supl.1-20200354. 
CJ. Menezes, J.A., et al.(2020).A contação de histórias no instagram como tecnologia leve em tempos pesados de pandemia. Psicol. Soc., Belo Horizonte , v. 32, 2020. https://doi.org/10.1590/1807-0310/2020v32240330.

CK. Pereira, M.D., et al.(2020).A pandemia de COVID-19, o isolamento social, consequências na saúde mental e estratégias de enfrentamento: uma revisão integrativa. Research, Society and Development, v.9, ed.7. DOI: https://doi.org/10.33448/rsd-v9i7.4548

CL. Moreira, W.C., et al.(2020). adoecimento mental na população geral e em profissionais de saúde durante a COVID-19: scoping review. Texto Contexto Enferm., Florianópolis, v. 29.. http://dx.doi.org/10.1590/1980-265x-tce-2020-0215.

CM. Martinez, M., et al(2020) . COVID-19 y comportamiento psicológico: revisión sistemática de los efectos psicológicos de las pandemias del siglo XXI. Rev. Méd. Chile, Santiago, v. 148, n. 8, p. 1139-1154. http://dx.doi.org/10.4067/S0034-98872020000801139.

CN. Alvarez, P.E., et al (2020). COVID-19: médicos, gastroenterología y emociones. Rev Col Gastroenterol, Bogotá , v. 35, supl. 1, p. 64-68 . http://dx.doi.org/10.22516/25007440.546.

CO. Leiva, A.M. et al. (2020). Dimensión Psicosocial De La Pandemia: La Otra Cara Del COVID-19. Cienc. enferm., Concepción , v. 26, 10 . http://dx.doi.org/10.29393/ce26-3dpal60003.

CP. Malta, D., et al.(2020).Distanciamento social, sentimento de tristeza e estilos de vida da população brasileira durante a pandemia de COVID-19. Saúde em Debate, online. DOI:10.1590/SciELOPreprints.1371.

CQ. Johnson, M.C., SALETTI-CUESTA, L., \& Tumas, N.(2020). Emociones, preocupaciones y reflexiones frente a la pandemia del COVID-19 en Argentina. Ciênc. Saúde Coletiva, Rio de Janeiro, v. 25, ed. 1, p. 2447-2456. http://dx.doi.org/10.1590/1413-81232020256.1.10472020.

CR. Sousa, A.R de., et al (2020) . Emoções e estratégias de Coping de homens à pandemia da COVID-19 no Brasil. Texto \& Contexto - Enferm., Florianópolis , v. 29. http://dx.doi.org/10.1590/1980-265x-tce-2020-0248.

CS. Clementino, F.S., et al.(2020). Enfermagem na atenção às pessoas com COVID-19: desafios na atuação do sistema cofen/corens. Texto \& Contexto - Enferm., Florianópolis , v. 29. http://dx.doi.org/10.1590/1980-265x-tce-2020-0251.

CT. Bezerra, C.B, et al (2020). Impacto psicossocial do isolamento durante pandemia de COVID-19 na população brasileira: análise transversal preliminar. Saude Soc., São Paulo, v. 29, n. 4. https://doi.org/10.1590/s0104-12902020200412.

CU. Moreira, W., et al.(2020). Intervenções em saúde mental em tempos de COVID-19: scoping review. SciELO Preprints. DOI:10.1590/SciELOPreprints.1007.

CV. Reis, S.N., et al.(2020). Pandemic, social isolation and the importance of people-plant interaction. Ornam. Hortic., Viçosa , v. 26, n. 3, p. 399-412. .https://doi.org/10.1590/2447-536x.v26i3.2185.

CW. Barros, M.B.A., et al.(2020) . Relato de tristeza/depressão, nervosismo/ansiedade e problemas de sono na população adulta brasileira durante a pandemia de COVID-19. Epidemiol. Serv. Saúde, Brasília , v. 29, n. 4. https://doi.org/10.1590/s1679-49742020000400018.

CX. Paiano, M., et al.(2020). Saúde mental dos profissionais de saúde na China durante pandemia do novo coronavírus: revisão integrativa. Rev. Bras. Enferm. Brasília , v. 73, ed. 2. https://doi.org/10.1590/0034-7167-2020-0338.

CY. Schmidt, B., et al.(2020) Saúde mental e intervenções psicológicas diante da pandemia do novo coronavírus (COVID-19). Estud.Psicol. (Campinas), Campinas, v. 37. https://doi.org/10.1590/1982-0275202037e200063.

CZ. Souza, A.R., et al.(2020) . Sentimento e emoções de homens no enquadramento da doença COVID-19. Ciênc. Saúde Coletiva, Rio de Janeiro , v. 25, n. 9 , p. 3481-3491. https://doi.org/10.1590/1413-81232020259.18772020.

DA. FIOCRUZ - Fundação Oswaldo Cruz. (2020). Recomendações para o cuidado de crianças em situação de isolamento hospitalar. FIOCRUZ, online. Recuperado 3 maio. http://docs.bvsalud.org/biblioref/2020/04/1087528/cartilha_criancas_06_04.pdf

DB. Rocha, S.V., et al.(2020) A pandemia de COVID-19 e a saúde mental de idosos: possibilidades de atividade física por meio dos Exergames. Rev Bras Ativ Fis Saúde, v.25. DOI: 10.12820/rbafs.25e0142

DC. Melo, G.A.A., et al (2020). Benefícios da auriculoacupuntura em profissionais de enfermagem atuantes na COVID-19 à luz da Teoria do Conforto. Rev Esc. Anna Nery, Rio de Janeiro, v. 24. https://doi.org/10.1590/2177-9465-ean-2020-0311

DD. Perez, B., et al.(2020). Consecuencias psicológicas de la cuarentena y el aislamiento social durante la pandemia de COVID-19. Revista Cubana de Salud Pública, [S.1.], v. 46. Recuperado 3 Fev 2021 de:: http://www.revsaludpublica.sld.cu/index.php/spu/article/view/2488.

DE. Santos, G.C., et al.(2020). COVID-19 nas prisões: efeitos da pandemia sobre a saúde mental de mulheres privadas de liberdade. Rev Baiana Enferm, v. 34. DOI: http://dx.doi.org/10.18471/rbe.v34.38235

DF. Silva, H.G.N., et al (2020) Efeitos da pandemia no novo Coronavírus na saúde mental de indivíduos e coletividades. J. nurs. health, v. 10, n.4. Recuperado 4 Fev 2021 de : https://periodicos.ufpel.edu.br/ojs2/index.php/enfermagem/article/view/18677/11414.

DG. Serpa Jr., O. et al.(2020) Escrita, memória e cuidado - Testemunhos de trabalhadores de saúde na pandemia. Revista Latinoamericana de Psicopatologia Fundamental, v.23, ed.3, p.620-645. http:// dx.doi.org/10.1590/1415-4714.2020v23n3p620.11.

DH. Souza, J.B. et al. (2020).Estratégia musical para cuidar de discentes de Enfermagem: Experiência no enfrentamento da COVID-19. Rev Baiana Enferm. v.34. DOI 10.18471/rbe.v34.37097

DI. Barbosa, D.J., et al.(2020). Fatores de estresse nos profissionais de enfermagem no combate à pandemia da COVID-19: síntese de evidências. Com. Ciências Saúde , v.31, ed.1. DOI: https://doi.org/10.51723/ccs.v31iSupp1\%201.651 
DJ. Almaguer, C, et al (2020). Gestión de seguridad psicológica del personal sanitario en situaciones de emergencia por COVID-19 en el contexto hospitalario o de aislamiento. Revista Cubana de Enfermería, [S.1.], v. 36, n. 2. Recuperado 4 Fev 2021 de: http://www.revenfermeria.sld.cu/index.php/enf/article/view/3704.

DK. Cobián, M. et al.(2020). Intervención psicoterapéutica breve en estudiantes caribeños de Medicina con reacciones psicológicas por impacto ante la COVID19. MEDISAN, vol. 24, no. 5, p.823-835..Recuperado 4 fev 2021 de https://www.redalyc.org/articulo.oa?id=368464850005.

DL. Saidel, M.G.B., et al. Intervenções em saúde mental para profissionais de saúde frente a pandemia de Coronavírus . Revista Enfermagem UERJ, [S.1.], v. 28, p. e49923, maio 2020. ISSN 0104-3552. Disponível em: https://www.e-publicacoes.uerj.br/index.php/enfermagemuerj/article/view/49923/33860. Acesso em: 4 fevereiro 2021. doi:https://doi.org/10.12957/reuerj.2020.49923.

DM. Urzúa, A., et al.(2020). La Psicología en la prevención y manejo del COVID-19. Aportes desde la evidencia inicial. Terapia Psicológica, v. 38, n. 1, p. 103-118, 29 abr. 2020. Recuperado 4 fev 2021 de: https://teps.cl/index.php/teps/article/view/273.

DN. Organização Pan Americana de Saúde (OPAS). COVID-19(2020). Recommended Interventions in Mental Health and Psychosocial Support (MHPSS) during the Pandemic. Recuperado 4 fev 2021 de: https://www.paho.org/en/documents/COVID-19-recommended-interventions-mental-health-and-psychosocialsupport-mhpss-during.

DO. Trigueiro, R.L., et al(2020). COVID-19 pandemic: report on the use of auriculotherapy to optimize emergency workers' health. Rev Bras Enferm., v.73. doi: http://dx.doi.org/10.1590/0034-7167-2020-0507

DP. Oliveira, E.N., et al(2020). Projeto vida em quarentena: estratégia para promoção da saúde mental de enfermeiros diante da COVID-19. Enferm. Foco, v. 11, ed.1, p.162-167. Recuperado 4 fev 2021 de: http://biblioteca.cofen.gov.br/projeto-vida-em-quarentena-saude-mental-enfermeiros-COVID-19/.

DQ. Humerez, D.C., et al (2020) . Saúde mental dos profissionais de enfermagem do Brasil no contexto da pandemia COVID-19: ação do Conselho Federal de Enfermagem. Cogitare Enferm. v.25. http://dx.doi.org/10.5380/ ce.v25i0.74115.1.

DR. Martins, A.B.T., et al (2020). Sentimento de angústia e isolamento social de universitários da área da saúde durante a pandemia da COVID-19. Rev Bras Promoç Saúde, v. 33. DOI: 10.5020/18061230.2020.11444

DS. Ornel, F., et al(2020). Pandemia de medo e COVID-19: impacto na saúde mental e possíveis estratégias. Artigo debates in psychiatry - Ahead of print, Porto Alegre, online. Recuperado 15 maio 2020 de https://www.researchgate.net/profile/Felix_Kessler2/publication/340442412_Pandemia_de_medo_e_COVID19_impacto_na_saude_mental_e_possiveis_estrategias/links/5e893140299bf130797c84f4/Pandemia-de-medo-e-COVID-19-impacto-na-saude-mental-epossiveis-estrategias.pdf

DT. Raiol, R. (2020). A prática de exercícios físicos é fundamental para a saúde física e mental durante a Pandemia da COVID-19. Brazilian Journal of health Review,Curitiba, v. 3, n. 2, p. 2804-2813.DOI:10.34119/bjhrv3n2-124

DU. Diniz, L \& Malloy, F.(2020). Saúde mental na pandemia de COVID-19: considerações práticas multidisciplinares sobre cognição, emoção e comportamento. Artigo debates in psychiatry - Ahead of print, Minas Gerais, online. Recuperado 15 maio 2020 de: https://www.researchgate.net/profile/Tercio_Apolinario-Souza2/publication/341255949_Saude_mental_na_pandemia_de_COVID_-

19_consideracoes_praticas_multidisciplinares_sobre_cognicao_emocao_e_comportamento/links/5eb5cf74aa6fdcc1f1dcae6b5/Saude-mental-na-pandemia-deCOVID-19-consideracoes-praticas-multidisciplinares-sobre-cognicao-emocao-e-comportamento.pdf

DV. Schmidt, B., et al(2020) Saúde mental e atenção psicossocial na pandemia COVID-19. A quarentena na COVID-19: orientações e estratégias de cuidado. FIOCRUZ, online. Recuperado 15 maio 2021 de https://www.fiocruzbrasilia.fiocruz.br/wp-content/uploads/2020/04/Sa\%C3\%BAde-Mental-eAten\%C3\%A7\%C3\%A3o-Psicossocial-na-Pandemia-COVID-19-A-quarentena-na-COVID-19-orienta\%C3\%A7\%C3\%B5es-e-estrat\%C3\%A9gias-decuidado.pdf.

DW. Lima SO., et al (2020). Impactos no comportamento e na saúde mental de grupos vulneráveis em época de enfrentamento da infecção COVID-19: revisão narrativa. Revista Eletrônica Acervo Saúde, (46), e4006.https://doi.org/10.25248/reas.e4006.2020

DX. Silva, S.G (2020). Pandemia e afetações das emoções: reflexões sobre a realidade da COVID-19 no estado do Amapá. RBSE Revista Brasileira de Sociologia da Emoção, v. 19, n. 55.Recupoerado 10 Fev 2021 de https://grem-grei.org/wp-content/uploads/2020/05/8_Selma_RBSEv19n55abril2020_SuplEspecial_maio2020.pdf

DY. Prigol, A.C \& Santos, E,L. Saúde mental dos profissionais de enfermagem diante da pandemia COVID-19. Research, Society and Development, v. 9. DOI: http://dx.doi.org/10.33448/rsd-v9i9.7563

DZ. Neves, A., et al (2020). O impacto da pandemia de COVID-19 nas emoções dos trabalhadores da saúde e na aprendizagem profissional. Research, Society and Development..DOI: http://dx.doi.org/10.33448/rsd-v9i12.11367

EA. Souza, M, et al (2020). Os programas de educação socioemocional no contexto da pandemia de COVID-19. Congresso internacional de educação e tecnologia. UFG, 2020. Recuperado 13 Fev 2021 de https://cietenped.ufscar.br/submissao/index.php/2020/article/view/1778/1414

EB. Rodrigues, J.V.S \& Lins, A.C.A. (2020). Centro Universitário Metropolitano da Amazônia, Brasil. Possíveis impactos causados pela pandemia da COVID19 na saúde mental de crianças e o papel dos pais neste cenário. Research, Society and Development, v. 9.. DOI: http://dx.doi.org/10.33448/rsd-v9i8.6533

EC. Santos, W.A (2020). O impacto da pandemia da COVID -19 na saúde mental dos profissionais de saúde: revisão integrativa. Research, Society and Development, v. 9. DOI: http://dx.doi.org/10.33448/rsd-v9i8.5470

ED. Borloti., E., et al(2020). Saúde mental e intervenções psicológicas durante a pandemia de COVID-19: Um panorama . Revista Brasileira de Análise Do Comportamento. 2020, vol. 16. DOI: http://dx.doi.org/10.18542/rebac.v16i1.8885

EE. Amestoy, S.C., (2020). Inteligência emocional: habilidade relacional para o enfermeiro líder na linha de frente contra o novo Coronavírus. J. nurs. health.; v. 10. Recuperado 14 fev de file:///C:/Users/Carlos/Desktop/Downloads/18993-65321-1-PB.pdf 
EF. Miranda, T.S.A., et al (2020). Incidência dos casos de transtornos mentais durante a pandemia da COVID-19. Revista Eletrônica Acervo Científico v. 17.. DOI: https://doi.org/10.25248/reac.e4873.2020.

EG. Pimentel, A.S.G \& Silva, M.N.R (2020). Saúde psíquica em tempos de CoronaVirus. Pesquisa, Sociedade e Desenvolvimento , [S. 1.] , v. 9, n. 7, pág. e11973602. DOI: 10.33448 / rsd-v9i7.3602

EH. Alves, J.,et al (2020). IGT na Pandemia da COVID-19: Apoio psicológico on-line em grupos abertos. IGT - Instituto de Gestalt-Terapia e Atendimento Familiar. Recuperado 15 fev 2021 de http://www.igt.psc.br/ojs/viewarticle.php?id=725\&layout=html

EI. Macedo, N.N., et al(2020). Capítulo VIII Pandemia do COVID-19 e seus efeitos sobre a saúde mental da população ou profissionais da saúde. Cartilha: Construção do saber sobre COVID-19. João Pessoa: Editora do CCTA. Recuperado 16 fev 2021 de http://www.ccta.ufpb.br/editoraccta/contents/titulos/saude/construcao-do-saber-sobre-COVID-19-1/construcao-do-saber-sobre-COVID-19.pdf\#page=188

EJ. Mazzaia, M.C., et al (2020). Saúde mental dos profissionais que atuam com pessoas em situação de rua- Pandemia de COVID-19.Cartilha da UNIFESP: Comissão de residência multiprofissional. Recuperado 16 Fev 2021 de: http://www.abennacional.org.br/site/wp-content/uploads/2020/09/Cartilh_2_Sa\%C3\%BAde_mental_SIt_rua.pdf.

EK. Soare., et al(2020). O uso da música para regulação do estado de ânimo no período pós-COVID-19, p. 397-416. Revista Música, v. 20n. 2 -Dossiê Música em Quarentena Universidade de São Paulo. DOI 10.11606/rm.v20i2.180074

EL. Silva, M.O., et al (2020). Impactos psicológicos do retorno às atividades laborais durante a pandemia COVID-19 em uma Policlínica Regional de Saúde na Bahia: um relato de experiência. Research, Society and Development, [S. 1.], v. 9, n. 11, p. e2029119372. DOI: 10.33448/rsd-v9i11.9372.

EM. Silva, N., et al(2020). Psicologia Positiva e COVID-19: relato de experiência do projeto de extensão "LAPPOT na Quarentena". Cidadania em Ação: Revista de Extensão e Cultura, v. 4, n. 2, p. 40-55. DOI: 10.5965/259464124240.

EN.Gonçalvez, L., et al(2020). Saúde de mulheres de dois grupos de atividade física: estudo de acompanhamento durante a COVID19. Revista Brasileira de Atividade Física \& Saúde . DOI10.12820/rbafs.25e0144

EO. Melo, B.C., et al(2021). Meditação na redução do estresse em profissionais de saúde na pandemia do COVID-19: revisão narrativa. Rev. Ciências Saúde v.31, 2021. Recuperado 17 fev 2021 de http://www.escs.edu.br/revistaccs/index.php/comunicacaoemcienciasdasaude/article/view/728

EP. Souto, A.R.G., et al (2020). A importância de praticar a atenção plena durante o distanciamento social. Semana de Pesquisa do Centro Universitário Tiradentes - SEMPESq - Alagoas. Recuperado 17 fev 2021 de https://eventos.set.edu.br/al_sempesq/article/view/13948/6065

EQ. Zwielewski. G., et al (2020). Protocolos para tratamento psicológico em pandemias: as demandas em saúde mental produzidas pela COVID-19. Revista debates in psychiatry. Recuperado 18 Fev 2021 de http://www.hu.ufsc.br/setores/neuropsicologia/wp-content/uploads/sites/25/2015/02/Protocolos-psic-empandemias-covid-final.pdf

ER. Nabuco, G., et al (2020). O impacto da pandemia pela COVID-19 na saúde mental: qual é o papel da Atenção Primária à Saúde. Rev Bras Med Fam Comunidade. Rio de Janeiro. DOI: https://doi.org/10.5712/rbmfc15(42)2532

ES. Almeida, R.S., et al(2020). Pandemia de COVID-19: guia prático para promoção da saúde mental de crianças e adolescentes.Sociedade Brasileira de Pediatria. Residência Pediátrica Rio De Janeiro. DOI: 10.25060/residpediatr-2020.v10n2-318

ET. Garrido, G.G., \& Rodrigues, R.C. (2020). Restrição de contato social e saúde mental na pandemia: possíveis impactos das condicionantes sociais. Journal of Health and Biological Sciences: Revista de saúde e ciências biológicas. DOI: http://dx.doi.org/10.12662/2317-3076jhbs.v8i1.3325.p1-9.2020

EU. Dolabella, B.M., et al(2020). Saúde mental e atenção psicossocial na pandemia COVID-19: crianças na pandemia COVID-19. Rio de Janeiro: Fiocruz/CEPEDES 20 p. Cartilha. Recuperado $21 \mathrm{fev} 2021 \mathrm{de}$ https://www.arca.fiocruz.br/handle/icict/41182

EV. Ribeiro, E.G., et al .(2020). Saúde Mental e COVID-19. Saúde Mental na Perspectiva do Enfrentamento à COVID -19: Manejo das Consequências Relacionadas ao Isolamento Social. Rev. Enfermagem e Saúde Coletiva, Faculdade de São Paulo, FSP. Recuperado 21 fev 2021 de https://revesc.org/index.php/revesc/article/view/59

EW. Cruz, N.,et al(2020). Apoio psicossocial em tempos de COVID-19: experiências de novas estratégias de gestão e ajuda mútua no sul da Bahia, Brasil. APS EM REVISTA. https://doi.org/10.14295/aps.v2i2.94

EX. Claro, C., et al (2020). A música na lupa da psicologia face à pandemia: Covid19!. Revista INFAD de Psicología. International Journal of Developmental and Educational Psychology., v. 1, n. 1, p. 143-154.. https://doi.org/10.17060/ijodaep.2020.n1.v1.1770.

EY. Almeida, R., et al(2020). A prática de Mindfulness em profissionais de saúde em tempos de COVID-19: uma revisão sistemática. Revista Qualidade HC, online. Recuperado 23 fev 2021 de https://www.hcrp.usp.br/revistaqualidade/uploads/Artigos/283/283.pdf.

EZ. Silva, B.L., et al(2020). As habilidades socioemocionais necessárias ao enfrentamento da COVID-19. Saúde Coletiva (Barueri), v. 10, n. 54, p. 2627-2632. DOI: https://doi.org/10.36489/saudecoletiva.2020v10i54p2627-2632

FA. Barbosa,CC., et al(2020). Orientações para o cuidado e autocuidado em saúde mental para os trabalhadores da Fiocruz: Diante da pandemia da doença pelo SARS-CoV-2 (COVID-19). Arca Fiocruz. Recuperado 22 Fev 2021 de : https://www.arca.fiocruz.br/bitstream/icict/43377/2/cartilha_cogepe_saudemental_2020-05-14\%5B1\%5D.pdf.

FB. Orfão, N.H., et al(2020). COVID-19: estratégias de enfrentamento e comportamentos adaptativos pelos profissionais de saúde durante a pandemia. Revista de Epidemiologia e Controle de Infecção, v. 10, n. 4.. DOI: http://dx.doi.org/10.17058/reci.v10i4.15462

FC. Cruz, A.T.A \& Silva, J.M (2020). Diálogos virtuais como estratégia de apoio à saúde mental em contexto de isolamento social. Revista asas da palavra, v. 17, n. 2, p. 22-29. DOI: http://dx.doi.org/10.17648/asas.v17i2.2164 
FD. Antiporta, D.A \& Bruni, A. (2020). Emerging mental health challenges, strategies, and opportunities in the context of the COVID-19 pandemic: Perspectives from South American decision-makers. Revista Panamericana de Saud Pública, v. 44. doi: 10.26633/RPSP.2020.154.

FE. Dias, W.B., et al (2020). Impactos na saúde mental causados pela pandemia de sars-cov-2 e isolamento social: relato de experiência. Research, Society and Development, v. 9, n. 8, p. e979986876-e979986876. DOI: https://doi.org/10.33448/rsd-v9i8.6876

FF. El Kadri, M.R \& Nazareth, C. (2020). Mitigação de efeitos psicossociais na pandemia COVID-19, Governo do Estado do Amazonas. Recuperado 22 fev 2021 de: http://www.sedecti.am.gov.br/wp-content/uploads/2021/01/mitigacao_de_efeitos.pdf.

FG. Danzmann, P.S., et al. (2020). Atuação do psicólogo na saúde mental da população diante da pandemia. J. nurs. health.. DOI: HTTPS://DOI.ORG/10.15210/JONAH.V10I4.18945

FH. Oliveira, D.S., et al (2020). COVID-19: do enfrentamento ao fortalecimento de estratégias em saúde mental - Revisão narrativa. Health Residencies Journal v. 1 n.4.. DOI: https://doi.org/10.51723/hrj.v1i4.34

FI. Corrêa, C.A., et al (2020). Níveis de estresse, ansiedade, depressão e fatores associados durante a pandemia de COVID-19 em praticantes de Yoga. Rev Bras de Ativ Fis e Saúde, v.25, Piracicaba-SP. DOI: https://doi.org/10.12820/rbafs.25e0118

FJ.Rocha.,N.L., et al (2020). Construindo o Projeto Cuidadosamente: reflexão sobre a saúde mental dos graduandos de Enfermagem frente ao COVID-19. Rev. Saúde Col. UEFS, Feira de Santana, Vol. 10. DOI: http://dx.doi.org/10.13102/rscdauefs.v10i1.5153

FK. Figel, F.C., et al (2020). Reorganização da atenção à saúde mental na pandemia de COVID-19. Rev Sal Pub do Paraná. DOI: https://doi.org/10.32811/25954482-2020v3sup1p118

FL. Nascimento, S., et al (2020). Pandemia COVID-19 e Perturbação Mental: Breve Revisão da Literatura. Revista Portuguesa De Psiquiatria E Saúde Mental, v.6 67-76. https://doi.org/10.51338/rppsm.2020.v6.i2.146

FM. Parente. A, et al (2020). Saúde mental de crianças e seus cuidadores diante da pandemia da COVID-19. Health Residencies Journal. DOI: https://doi.org/10.51723/hrj.v1i5.14

FN. Santos, M.F.R \& Oliveira M.E.F (2020). Saúde mental em tempos de COVID-19: a importância do atendimento psicológico remoto. Revista Transformar $14^{\circ}$ edição. Recuperado 26 fev 2021 de http://www.fsj.edu.br/transformar/index.php/transformar/article/view/378 